\title{
Comparative efficacy and safety of Chinese herbal injections combined with the FOLFOX regimen for treating gastric cancer in China: a network meta-analysis
}

\author{
Dan Zhang ${ }^{1}$, Jiewen Zheng ${ }^{1}$, Mengwei $\mathrm{Ni}^{1}$, Jiarui $\mathrm{Wu}^{1}{ }^{1}$ Kuaihuan Wang ${ }^{1}$, Xiaojiao \\ Duan $^{1}$, Xiaomeng Zhang ${ }^{1}$ and Bing Zhang ${ }^{1}$ \\ ${ }^{1}$ Department of Clinical Chinese Pharmacy, School of Chinese Materia Medica, Beijing University of Chinese Medicine, Beijing \\ 100102, China
}

Correspondence to: Jiarui Wu, email: exogamy@163.com

Keywords: Chinese herbal injections, FOLFOX regimen, gastric cancer, network meta-analysis

Received: May 26, 2017 Accepted: August 04, $2017 \quad$ Published: August 18, 2017

Copyright: Zhang et al. This is an open-access article distributed under the terms of the Creative Commons Attribution License 3.0 (CC BY

3.0), which permits unrestricted use, distribution, and reproduction in any medium, provided the original author and source are credited.

\section{ABSTRACT}

Background: Chinese herbal injections (CHIs) have been proven beneficial to patients with gastric cancer for improving clinical efficacy and relieving adverse reactions (ADRs) of chemotherapy. A network meta-analysis (NMA) was conducted in this study to assess the comparative efficacy and safety of CHIs combined with FOLFOX regimen for treating gastric cancer.

Results: A total of 2316 records were searched, and 81 eligible RCTs involving 15 types of CHIs and 5978 patients were included in the NMA. The results showed that patients who received Shengqifuzheng+ FOLFOX, Compound kushen+ FOLFOX, Huachansu+ FOLFOX, Astragalus+ FOLFOX, Kangai+ FOLFOX, and Lentinan injection + FOLFOX could significantly improve clinical efficacy than using FOLFOX single, and their odds ratios (OR) and 95\% confidence intervals (CI)s were $1.57(1.19,2.09), 2.12$ $(1.62,2.78), 1.72(1.08,2.80), 3.06(1.01,8.99), 2.01(1.52,2.70)$, and $1.99(1.20,3.38)$ respectively. Furthermore, the therapy of Aidi+ FOLFOX, Shenqifuzheng+ FOLFOX, Compound Kushen+ FOLFOX, Huachansu+ FOLFOX, Astragalus polysaccharides+ FOLFOX, Kangai+ FOLFOX, Ginseng polysaccharide+ FOLFOX, Lentinan+ FOLFOX, Xiaoaiping+ FOLFOX, and Shenmai injection + FOLFOX could also achieve a higher performance status compared with FOLFOX regimen alone. Similarly, patients who received CHIs combine with FOLFOX regimen were associated with a significantly decrease the incidence of leucopenia, gastrointestinal reaction and hepatic dysfunction. Cluster analysis demonstrated that Astragalus polysaccharides+ FOLFOX, and Kangai+ FOLFOX seemed optimal therapies in improving clinical efficacy and performance status; Astragalus polysaccharides+ FOLFOX was superior in reducing leucopenia and gastrointestinal reaction; Disodium Cantharidinate and Vitamin B6+ FOLFOX was associated with favorable effects in reducing gastrointestinal reaction and hepatic dysfunction. By contrary, receiving FOLFOX regimen single was proved to rank the worst for these outcomes.

Materials and Methods: A comprehensive literature search was performed in several electronic databases to identify randomized controlled trial (RCTs) regarding CHIs for gastric cancer until January 10, 2017. The quality assessment was accomplished according to the Cochrane risk of bias tool and the methodological section of the CONSORT statement. And a random-effects model NMA was utilized to compare different CHIs combined with FOLFOX regimen with regard to efficacy and safety. Data were analyzed using STATA 12.0 and Win-BUGS 1.4 software. 


\begin{abstract}
Conclusions: The results of this NMA suggested that among 15 types of CHIs, Astragalus polysaccharides injection combined with FOLFOX regimen seemed optimal for patients with gastric cancer in improving clinical efficacy and performance status, and relieving ADRs. However, our findings should be confirmed by more prospectively designed, large-sample and multi-center RCTs.
\end{abstract}

\section{INTRODUCTION}

Gastric cancer is a leading cause of cancer-related deaths worldwide; its morbidity is in the first place of gastrointestinal malignancies [1-3]. Currently, gastric cancer has the characteristics of high morbidity and mortality, low early diagnosis rate, surgical resection rate and 5 years survival rate [4-6]. The etiology of gastric cancer has not yet been elucidated, whereas it is reported that the development of gastric cancer maybe related with chemical carcinogen, micronutrient deficiency, microbial infection, hereditary and other factors [7]. Surgical treatment is the therapeutic modality that offers the greatest possibility of cure for patients with gastric cancer, besides, chemotherapy and radiotherapy are important therapeutic options for patients who are suffering from distant metastases or unable to receive surgery [8-9]. FOLFOX regimen is one of the internationally recognized first-line chemotherapy regimen for gastric cancer, and this regimen is composed of 5-fluorouracil (5-Fu), leucovorin (LV) and oxaliplatin (L-OHP) [7, 10]. According to the relevant studies, L-OHP and 5-Fu may cause ADRs such as peripheral neurotoxicity, pancytopenia and severe gastrointestinal toxicity $[11,12]$. It has been thousands of years that traditional Chinese medicine (TCM) are applied at clinical to treating cancers in China, Japan, and other Asian countries [13-14]. As complementary and alternative medicine, TCM has become one of main methods for cancer comprehensive treatment, because it has the advantages of improving the clinical symptoms, enhancing immunity and alleviating chemotherapyinduced ADRs $[15,16]$. Meanwhile, TCM could have therapeutic effects in toxicity reducing and efficacy enhancing when it is in combination with radiotherapy or chemotherapy [17]. The results of a network pharmacological study present that 13 Chinese herbs were associated with survival benefit for patients with stage IV gastric cancer through correlation analysis [18]. Based on an orthotopic mouse model of human gastric cancer, the pharmacological study confirms that ginsenoside Rg3 could and reduce lymphatic metastasis and inhibit tumor growth, those may be related to suppress expression of vascular endothelial growth factor receptor-C, lymphogeneous metastasis and vascular endothelial growth factor-D [19]. Another pharmacological study reveals that the compounds which isolated from Sophora Flavescens could induce mitochondria-mediated apoptosis in human gastric cancer cells, the possible mechanisms might be the induction of cell cycle arrest and apoptosis
[20]. And through the nationwide survey in China, the study indicates that $42.4 \%$ of 51,382 cancer patients use 33 anticancer Chinese patent medicines and 24.8\% of the cancer patients used both anticancer Chinese patent medicines and anticancer western medicines [21]. Furthermore, CHIs possess the advantages of high bioavailability, high curative effects compared with TCM decoction [22].

Although NMA is developed from the standard meta-analysis, it allows for the simultaneous evaluation of multiple interventions to provide more comprehensive and valuable information for clinical decision-making through both direct and indirect comparisons [23-25]. Moreover, NMA could sort the different interventions based on their therapeutic effects and the probability of optimal interventions [24-25]. Given the lack of headto-head RCTs between different CHIs, a NMA was conducted to compare the efficacy and safety of multiple CHIs combined with FOLFOX regimen simultaneously to investigate which is the best $\mathrm{CHI}$ for gastric cancer.

\section{RESULTS}

\section{Literature search and baseline characteristics}

As illustrated by the PRISMAflow diagram (Figure 1), a total of 2316 suitable articles were identified via a primary search of the aforementioned literature databases. According to inclusion and exclusion criteria, the appropriate trails were selected strictly. Finally, 81 eligible RCTs involving 15 types of CHIs and 5978 patients were included in the NMA, and the included RCTs were all published in Chinese [26-106]. 15 types of CHIs were identified, including Astragalus injection (1 trial), Shenmai injection (1 trial), Disodium cantharidinate and vitamin B6 injection (1 trial), Delisheng injection (1 trial), Elemene injection (1 trial), Placental peptide injection (1 trial), Xiaoaiping injection (2 trial), Ginseng polysaccharide injection (2 trial), Astragalus polysaccharide injection (2 triasl), Lentinan injection (5 trials), Hauchansu injection (6 trials), Aidi injection (9 trials), Kangai injection (14 trials), Compound kushen injection (17 trials), Shenqifuzheng injection (18 trials).

The 81 RCTs included 15 types of CHIs and 5978 patients with gastric cancer, among 3049 patients were in CHIs group and 2929 patients were in FOLFOX groups. All of the included RCTs reported patient numbers and ages. Moreover, 74 (91.36\%), 52 (64.20\%), 49 (60.49\%) and $56(69.14 \%)$ trials respectively described the patients' 
gender, tumor staging, expected survival time and Karnofsky performance score (KPS) before treatment. More details of baseline characteristics for individual trials were presented in Supplementary Table 1. Besides, the network graph revealing the distribution of RCTs and relationship of CHIs for 5 outcomes was depicted in Figure 2.

\section{Results of quality assessment}

The results of quality assessment for all included RCTs were showed in Supplementary Table 2. Although all of the included RCTs mentioned randomization and described the inclusion and exclusion criteria, the calculation of sample size was not mentioned in each RCT. In terms of random sequence generation methods, 11 RCTs $(13.58 \%)$ used a random number table, random lottery form was adopted to divide into groups in 1 trail $(1.23 \%), 1$ RCT $(1.23 \%)$ adopted the method of oddeven number, and 2 RCTs $(2.47 \%)$ applied the method of hospitalized time difference. Moreover, there were only 3 RCTs mentioning blinding method. In addition, 6 RCTs (7.41\%), 8 RCTs $(9.88 \%), 63$ RCTs $(77.78 \%)$ and 21RCTs
(25.93\%) provided the details about funding supports, follow-up, ADRs, and medical ethics.

\section{Outcomes}

\section{The clinical efficacy}

The data of clinical efficacy were available for 61 RCTs involving 10 CHIs. The results based on NMA demonstrated that patients who received Shengqifuzheng+ FOLFOX $(\mathrm{OR}=1.57,95 \%$ CI $=1.19-2.09)$, Compound kushen+ FOLFOX (OR $=2.12,95 \%$ $\mathrm{CI}=1.62-2.78)$, Huachansu + FOLFOX $(\mathrm{OR}=1.72,95 \%$ $\mathrm{CI}=1.08-2.80)$, Astragalus + FOLFOX $(\mathrm{OR}=3.06,95 \%$ $\mathrm{CI}=1.01-8.99)$, Kangai + FOLFOX $(\mathrm{OR}=2.01,95 \%$ $\mathrm{CI}=1.52-2.70)$, and Lentinan injection + FOLFOX $(\mathrm{OR}=1.99,95 \% \mathrm{CI}=1.20-3.38)$ could significantly improve clinical efficacy than using FOLFOX single. Besides, on the basis of combining with FOLFOX regimen, there was without significantly difference between different CHIs. The OR along with 95\% CI of each intervention for clinical efficacy was presented in Table 1. According to the calculated probabilities for

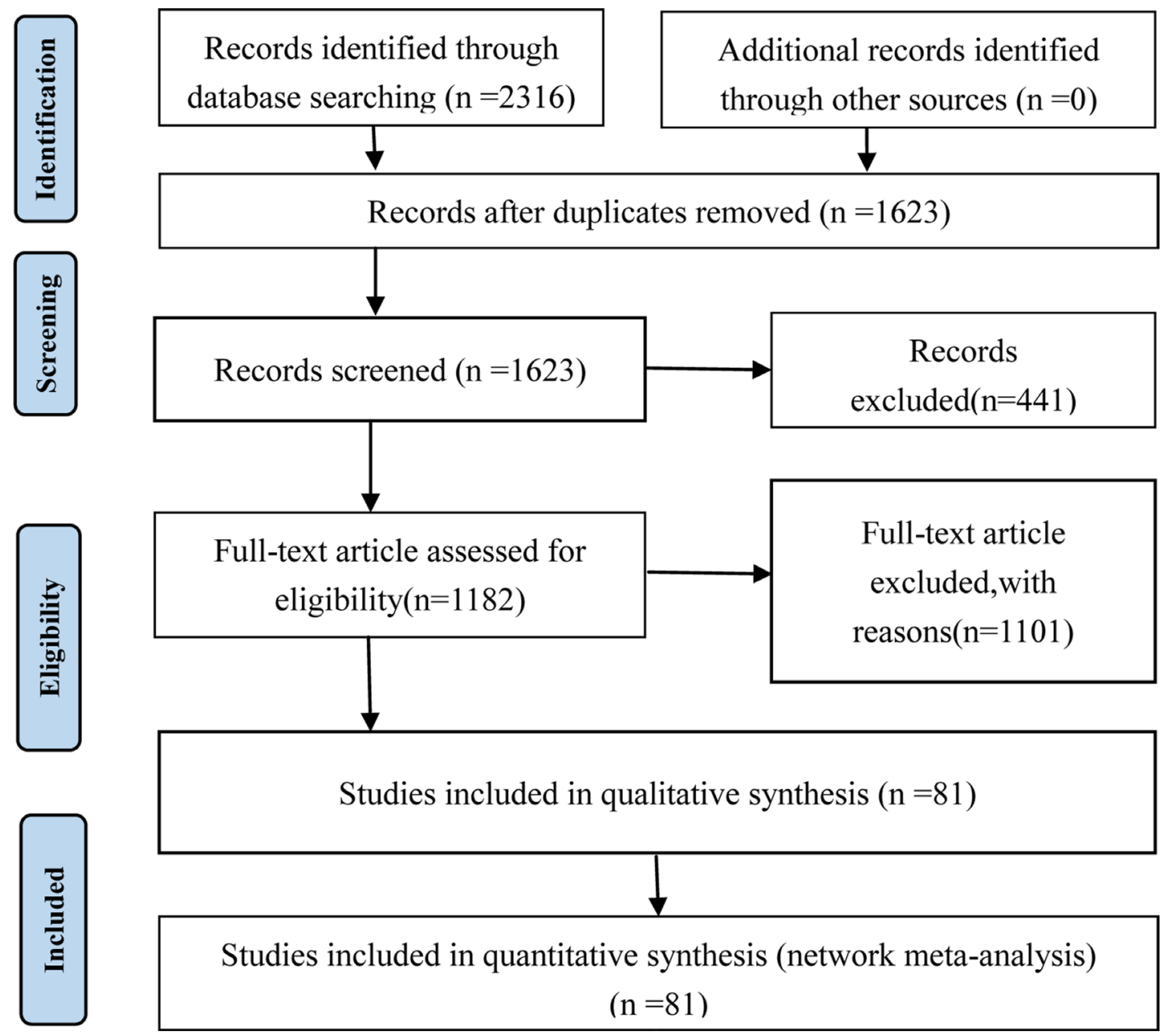

Figure 1: PRISMA flow diagram. 
Table 1: Network meta-analysis results of clinical efficacy (upper right quarter) and performance status (lower left quarter)

\begin{tabular}{|c|c|c|c|c|c|c|c|c|c|c|c|c|}
\hline $\begin{array}{l}\text { AD } \\
+ \text { FOLFOX }\end{array}$ & $\begin{array}{l}0.70 \\
(0.49,1.01)\end{array}$ & $1.11(0.69,1.75)$ & $1.50(0.94,2.32)$ & $1.23(0.67,2.18)$ & $1.35(0.49,3.95)$ & $2.16(0.71,6.68)$ & $1.42(0.88,2.25)$ & $0.89(0.40,2.03)$ & $1.41(0.74,2.69)$ & $0.98(0.44,2.26)$ & - & -- \\
\hline $0.42(0.24,0.75)$ & FOLFOX & $1.57(1.19,2.09)$ & $2.12(1.62,2.78)$ & $1.72(1.08,2.80)$ & $1.92(0.72,5.24)$ & $3.057(1.01,8.99)$ & $2.01(1.52,2.70)$ & $1.26(0.61,2.62)$ & $1.99(1.20,3.38)$ & $1.40(0.68,2.87)$ & -- & -- \\
\hline $1.27(0.63,2.51)$ & $2.99(2.06,4.34)$ & $\begin{array}{l}\text { SQFZ } \\
+ \text { FOLFOX }\end{array}$ & $1.35(0.90,2.00)$ & $1.11(0.63,1.95)$ & $1.23(0.44,3.47)$ & $1.97(0.63,5.96)$ & $1.28(0.84,1.91)$ & $0.80(0.37,1.76)$ & $1.28(0.72,2.34)$ & $0.89(0.41,1.95)$ & -- & -- \\
\hline $1.16(0.56,2.38)$ & $2.74(1.77,4.22)$ & $0.92(0.51,1.63)$ & $\begin{array}{l}\text { CKS } \\
+ \text { + FOLFOX }\end{array}$ & $0.81(0.47,1.44)$ & $0.91(0.33,2.60)$ & $1.44(0.46,4.44)$ & $0.94(0.64,1.42)$ & $0.59(0.27,1.32)$ & $0.94(0.53,1.73)$ & $0.66(0.31,1.43)$ & -- & -- \\
\hline $1.24(0.46,3.43)$ & $2.94(1.32,6.77)$ & $0.98(0.41,2.48)$ & $\begin{array}{l}1.072 \\
(0.43,2.77)\end{array}$ & $\begin{array}{l}\text { HCS } \\
+ \text { FOLFOX }\end{array}$ & $1.11(0.36,3.38)$ & $1.77(0.53,5.87)$ & $1.16(0.66,2.00)$ & $0.73(0.31,1.70)$ & $1.15(0.58,2.38)$ & $0.80(0.34,1.91)$ & -- & -- \\
\hline $4.81(1.64,14.91)$ & $11.38(4.62,30.17)$ & $\begin{array}{l}3.82 \\
(1.44,10.85)\end{array}$ & $\begin{array}{l}4.16 \\
(1.53,12.08)\end{array}$ & $3.89(1.13,13.6)$ & $\begin{array}{l}\text { AP } \\
+ \text { + FOLFOX }\end{array}$ & $1.55(0.35,7.05)$ & $1.037(0.37,2.81)$ & $0.66(0.19,2.42)$ & $1.037(0.34,3.10)$ & $0.72(0.21,2.44)$ & -- & -- \\
\hline - & - & - & - & - & - & $\begin{array}{l}\text { AI } \\
+ \text { FOLFOX }\end{array}$ & $0.66(0.22,2.02)$ & $0.42(0.11,1.45)$ & $0.65(0.20,2.24)$ & $0.46(0.12,1.71)$ & -- & -- \\
\hline $1.82(0.90,3.72)$ & $4.30(2.91,6.50)$ & $1.44(0.84,2.51)$ & $1.57(0.88,2.87)$ & $1.46(0.58,3.61)$ & $0.38(0.13,1.02)$ & - & \begin{tabular}{|l} 
KA \\
+ FOLFOX
\end{tabular} & $0.63(0.29,1.39)$ & $0.99(0.56,1.83)$ & $0.70(0.32,1.51)$ & -- & -- \\
\hline $1.93(0.66,5.78)$ & $4.55(1.87,11.71)$ & $1.52(0.58,4.23)$ & $1.66(0.62,4.70)$ & $1.56(0.45,5.33)$ & $0.40(0.11,1.49)$ & - & $1.06(0.39,2.92)$ & $\begin{array}{l}\text { GP } \\
+ \text { FOLFOX }\end{array}$ & $1.59(0.63,3.97)$ & $1.10(0.39,3.15)$ & -- & -- \\
\hline $1.36(0.61,3.09)$ & $3.23(1.88,5.66)$ & $\begin{array}{l}1.082 \\
(0.56,2.12)\end{array}$ & $1.18(0.59,2.42)$ & $1.10(0.40,2.94)$ & $0.28(0.094,0.82)$ & - & $0.75(0.38,1.48)$ & $0.71(0.24,2.05)$ & $\begin{array}{l}\text { LE } \\
\text { + FOLFOX }\end{array}$ & $0.70(0.29,1.74)$ & -- & -- \\
\hline $1.37(0.48,3.92)$ & $3.23(1.36,7.85)$ & $\begin{array}{l}1.085 \\
(0.42,2.84)\end{array}$ & $1.18(0.45,3.17)$ & $1.096(0.33,3.64)$ & $0.28(0.079,1.01)$ & - & $0.75(0.29,1.96)$ & $0.71(0.20,2.51)$ & $1.00(0.36,2.82)$ & \begin{tabular}{|l} 
XAP \\
+ + FOLFOX
\end{tabular} & - & -- \\
\hline $1.74(0.50,6.39)$ & $4.11(1.34,13.33)$ & $0.73(0.21,2.35)$ & $0.67(0.19,2.19)$ & $0.72(0.17,2.90)$ & $2.77(0.63,12.16)$ & - & $1.05(0.31,3.47)$ & $1.11(0.25,4.76)$ & $0.78(0.22,2.75)$ & $0.79(0.18,3.29)$ & $\begin{array}{l}\text { SM } \\
+ \\
\text { FOLFOX }\end{array}$ & -- \\
\hline $1.23(0.28,5.94)$ & $2.90(0.73,12.68)$ & $0.97(0.23,4.45)$ & $\begin{array}{l}1.057 \\
(0.25,4.91)\end{array}$ & $0.98(0.20,5.19)$ & $0.25(0.048,1.42)$ & - & $0.67(0.16,3.10)$ & $1.57(0.29,8.19)$ & $1.12(0.23,4.91)$ & $1.12(0.20,5.78)$ & $\begin{array}{l}0.71 \\
(0.12,4.48)\end{array}$ & $\begin{array}{l}\text { EL } \\
\text { +FOLFOX }\end{array}$ \\
\hline
\end{tabular}

clinical efficacy, the 10 types of CHIs were ranked as follows: Astragalus $(83.45 \%, 1$ trail $)>$ Compound kushen $(74.4 \%, 13$ trails $)>$ Kangai $(68.65 \%, 12$ trails $)>$ Lentinan $(65.87 \%, 4$ trails $)>$ Astragalus polysaccharides $(59.76 \%$, 1 trails $)>$ Huachansu $(53.01 \%, 6$ trails $)>$ Shenqifuzheng $(42.14 \%, 13$ trails $)>$ Xiaoaiping $(35.66 \%, 2$ trails $)>$ Aidi $(33.24 \%, 7$ trails $)>$ Ginseng polysaccharide $(27.72 \%, 2$ trails). The rank of cumulative probabilities for clinical efficacy was revealed in Figure 3A.

\section{Performance status}

A total of $45 \mathrm{RCTs}$ with $11 \mathrm{CHIs}$ contributed to performance status analysis. The results indicated that the therapies of Aidi +FOLFOX (OR $=0.42,95 \% \mathrm{CI}=$ $0.24-0.75)$, Shenqifuzheng +FOLFOX (OR $=2.99,95 \%$ $\mathrm{CI}=2.06-4.34)$, Compound Kushen +FOLFOX (OR = $2.74,95 \% \mathrm{CI}=1.77-4.22)$, Huachansu +FOLFOX (OR $=2.94,95 \% \mathrm{CI}=1.32-6.77)$, Astragalus polysaccharides + FOLFOX $(\mathrm{OR}=11.38,95 \% \mathrm{CI}=4.62-30.17)$, Kangai +FOLFOX $(\mathrm{OR}=4.30,95 \% \mathrm{CI}=2.91-6.50)$, Ginseng polysaccharide +FOLFOX (OR $=4.55,95 \%$ $\mathrm{CI}=1.87-11.71)$, Lentinan +FOLFOX $(\mathrm{OR}=3.23,95 \%$ $\mathrm{CI}=1.88-5.66)$, Xiaoaiping +FOLFOX $(\mathrm{OR}=3.23$, $95 \% \mathrm{CI}=1.36-7.85)$, Shenmai +FOLFOX $(\mathrm{OR}=4.11$, $95 \% \mathrm{CI}=1.34-13.33$ ) were associated with favorable responses in terms of performance status compared with using FOLFOX regimen single, and significant difference was detected between these groups. Moreover, on the basis of combining with FOLFOX regimen, Astragalus polysaccharides was proven to significantly improve performance status than Aidi $(\mathrm{OR}=4.81,95 \%$ $\mathrm{CI}=1.64-14.91)$, Shenqifuzheng $(\mathrm{OR}=3.82,95 \%$ $\mathrm{CI}=1.44-10.85)$, Compound kushen $(\mathrm{OR}=4.16,95 \%$
$\mathrm{CI}=1.53-12.08)$, Huachansu $(\mathrm{OR}=3.89,95 \% \mathrm{CI}=$ 1.13-13.6), Lentinan $(\mathrm{OR}=0.28,95 \% \mathrm{CI}=0.094$ 0.82 ), and the difference between these CHIs groups was statistically significant (Table 1). As the Figure 3B displayed, the rankings of 11 CHIs based on their SUCRA value were: Astragalus polysaccharides $(97.12 \%, 2$ trails ) $>$ Kangai $(71.23 \%, 8$ trails $)>$ Ginseng polysaccharide $(69.38 \%, 2$ trails $)>$ Shenmai $(62.5 \%, 1$ trail $)>$ Lentinan $(50.09 \%, 5$ trails $)>$ Xiaoaiping $(50.07 \%, 2$ trails $)>$ Elemene $(44.85 \%, 1$ trail $)>$ Huachansu $(44.11 \%, 3$ trails $)$ $>$ Shenqifuzheng $(43.94 \%, 10$ trails $)>$ Compound kushen $(37.26 \%, 7$ trails $)>$ Aidi $(28.68 \%, 4$ trails $)$.

\section{ADRs}

\section{Leucopenia}

A total of $35 \mathrm{RCTs}$ involving $12 \mathrm{CHIs}$ provided data for leucopenia, the results revealed that: the therapies of Aidi +FOLFOX (OR $=3.64,95 \% \mathrm{CI}=2.03-6.65)$, Shenqifuzheng +FOLFOX $(\mathrm{OR}=0.51,95 \% \mathrm{CI}=0.28$ $0.88)$, Compound kushen +FOLFOX (OR $=0.33,95 \%$ $\mathrm{CI}=0.21-0.49)$, Huachansu + FOLFOX $(\mathrm{OR}=0.28,95 \%$ $\mathrm{CI}=0.099-0.77)$, Astragalus polysaccharides + FOLFOX $(\mathrm{OR}=0.22,95 \% \mathrm{CI}=0.060-0.74)$, Kangai + FOLFOX $(\mathrm{OR}=0.22,95 \% \mathrm{CI}=0.12-0.37)$, Lentinan +FOLFOX $(\mathrm{OR}=0.26,95 \% \mathrm{CI}=0.069-0.94)$ clearly stood better with regard to relieve leucopenia than only receiving FOLFOX regimen, with significant differences between these groups. Among CHIs groups, Kangai +FOLFOX yielded a better result for reducing leucopenia compared with Shenqifuzheng +FOLFOX (OR $=0.43,95 \%$ $\mathrm{CI}=0.19-0.94)$, and there was significant difference between two groups. The results of each intervention 
for leucopenia were provided in Table 2. According to cumulative probabilities for leucopenia in Figure 4A, 12 types of CHIs were ranked as follows: Kangai (77.91\%, 6 trails) $>$ Astragalus polysaccharides $(72.32 \%, 1$ trails $)$ $>$ Aidi $(64.23 \%, 5$ trails $)>$ Lentinan $(63.02 \%, 1$ trails $)>$ Hauchansu $(61.36 \%, 2$ trails $)>$ Disodium cantharidinate and vitamin B6 $(59.66 \%, 1$ trail $)>$ Compound kushen $(53.12 \%, 9$ trails $)>$ Placenta polypeptide $(51.72 \%, 1$ trail) $>$ Shenmai $(45.21 \%, 1$ trail $)>$ Xiaoaiping $(36.56 \%$, 2 trails $)>$ Delisheng $(36.52 \%, 1$ trail $)>$ Shenqifuzheng (26.47\%, 5 trails).

\section{Gastrointestinal reaction}

57 eligible RCTs enrolling 14 types of CHIs reported the data of gastrointestinal reaction. In respect of gastrointestinal reaction, the results between comparisons were revealed in Table 2. As combination therapies, Aidi +FOLFOX (OR $=2.74,95 \% \mathrm{CI}=1.81-4.18)$, Disodium cantharidinate and vitamin B6 +FOLFOX $(\mathrm{OR}=0.30,95 \% \mathrm{CI}=0.082-0.98)$, Shenmai + FOLFOX $(\mathrm{OR}=0.28,95 \% \mathrm{CI}=0.083-0.80)$, Shenqifuzheng + FOLFOX $(\mathrm{OR}=0.37,95 \% \mathrm{CI}=0.26-0.52)$, Delisheng + FOLFOX $(\mathrm{OR}=0.37,95 \% \mathrm{CI}=0.15-0.86)$, Compound kushen +FOLFOX $(\mathrm{OR}=0.45,95 \% \mathrm{CI}=0.32-0.64)$, Huachansu +FOLFOX $(\mathrm{OR}=0.35,95 \% \mathrm{CI}=0.19-0.64)$, Astragalus polysaccharides + FOLFOX $(\mathrm{OR}=0.16,95 \%$ $\mathrm{CI}=0.049-0.50)$, Kangai +FOLFOX (OR $=0.43$, $95 \%$ CI $=0.31-0.6)$, Placenta polypeptide + FOLFOX
$(\mathrm{OR}=0.29,95 \% \mathrm{CI}=0.082-0.94)$, Lentinan + FOLFOX $(\mathrm{OR}=0.40,95 \% \mathrm{CI}=0.21-0.74)$ demonstrated significant superiority in relieving gastrointestinal reaction over FOLFOX regimen, and there were significant differences between these groups. Additionally, there was no significant difference between different CHIs groups in respect of gastrointestinal reaction. Given the cumulative probabilities for gastrointestinal reaction as presented in Figure 4B, 14 types of CHIs ranked as follows: Astragalus polysaccharides $(88.56 \%, 1$ trail $)>$ Shenmai $(68.16 \%, 1$ trail $)>$ Placenta polypeptide $(65.5 \%, 1$ trail $)$ $>$ Disodium cantharidinate and vitamin B6 (63.99\%, 1 trail) $>$ Huachansu $(58.79 \%, 4$ trails $)>$ Shenqifuzheng $(56.41 \%, 12$ trails $)>$ Aidi $(56.29 \%, 8$ trails $)>$ Delisheng $(54.7 \%, 1$ trail $)>$ Lentinan $(48.95 \%, 4$ trails $)>$ Elemene $(42.77 \%, 1$ trail $)>$ Kangai $(41.83 \%, 12$ trails $)>$ Ginseng polysacchride $(39.72 \%, 1$ trail) $>$ Compound kushen (38.05\%, 9 trails) $>$ Xiaoaiping $(22.15 \%, 1$ trails $)$.

\section{Hepatic dysfunction}

19 RCTs including 9 types of CHIs provided sufficient data for estimating hepatic dysfunction. The results showed that the combined therapies of Aidi +FOLFOX 4.38 (1.76,11.08), Shenqifuzheng +FOLFOX 0.15 (0.014,0.94), Compound kushen +FOLFOX 0.21 $(0.11,0.38)$, Kangai +FOLFOX $0.34(0.14,0.82)$ had significant benefits of relieving hepatic dysfunction compared with FOLFOX regimen, and statistical
A

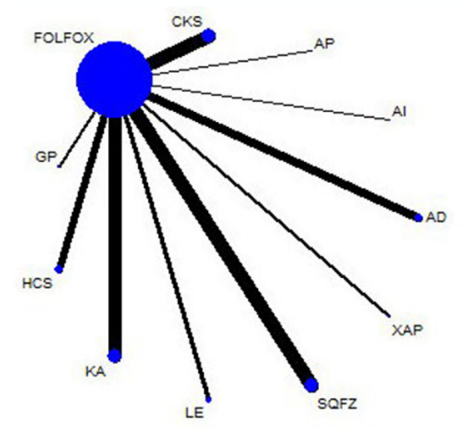

B

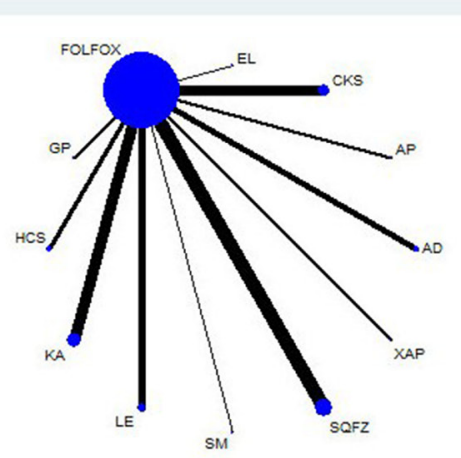

C

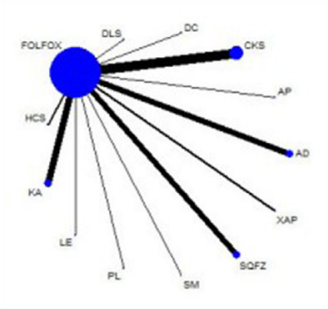

D

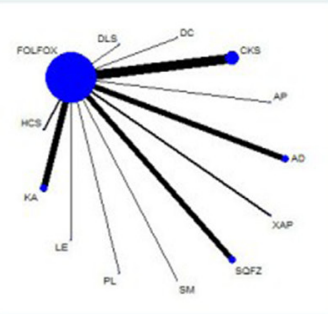

E

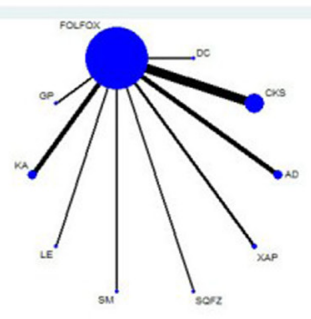

Figure 2: Network graph for 5 outcomes. Note: (A) Clinical efficacy; (B) Performance status; (C) Leucopenia; (D) Gastrointestinal reaction; (E) Hepatic dysfunction; FOLFOX: FOLFOX Chemotherapy regimen; CKS: Compound kushen injection; AP: Astragalus polysaccharide injection; AI: Astragalus injection; AD: Aidi injection; XAP: Xiaoaiping injection; SQFZ: Shenqifuzheng injection; LE: Lentinan injection; KA: Kangai injection; HCS: Huachansu injection; GP: Ginseng Polysacchride Injection; EL: Elemene injection; SM: Shenmai injection; DLS: Delisheng injection; DC: Disodium cantharidinate and vitamin B6 injection; PL: Placenta polypeptide injection. 
Table 2: Results of the network meta-analysis for leukopenia (upper right quarter), gastrointestinal reaction (lower left quarter)

\begin{tabular}{|c|c|c|c|c|c|c|c|c|c|c|c|c|c|c|}
\hline $\begin{array}{l}\text { AD } \\
\text { +FOLFOX }\end{array}$ & \begin{tabular}{|l}
3.64 \\
$(2.03,6.65)$
\end{tabular} & $\begin{array}{l}1.028 \\
(0.22,4.54)\end{array}$ & $\begin{array}{l}1.38 \\
(0.40,4.71)\end{array}$ & $\begin{array}{l}1.86 \\
(0.81,4.14)\end{array}$ & $\begin{array}{l}1.61 \\
(0.51,5.10)\end{array}$ & $\begin{array}{l}1.18 \\
(0.57,2.47)\end{array}$ & $\begin{array}{l}1.022 \\
(0.31,3.35)\end{array}$ & $\begin{array}{l}0.79 \\
(0.19,3.13)\end{array}$ & $\begin{array}{l}0.80 \\
(0.35,1.77)\end{array}$ & $\begin{array}{l}1.23 \\
(0.30,4.68)\end{array}$ & $\begin{array}{l}0.95 \\
(0.23,4.01)\end{array}$ & $\begin{array}{l}1.61 \\
(0.54,4.61)\end{array}$ & -- & -- \\
\hline $\begin{array}{l}2.74 \\
(1.81,4.18)\end{array}$ & FOLFOX & $\begin{array}{l}0.28 \\
(0.068,1.08)\end{array}$ & $\begin{array}{l}0.38 \\
(0.13,1.10)\end{array}$ & $\begin{array}{l}0.51 \\
(0.28,0.88)\end{array}$ & $\begin{array}{l}0.44 \\
(0.16,1.18)\end{array}$ & \begin{tabular}{|l}
0.33 \\
$(0.21,0.49)$
\end{tabular} & \begin{tabular}{|l}
0.28 \\
$(0.099,0.77)$
\end{tabular} & \begin{tabular}{|l}
0.22 \\
$(0.060,0.74)$
\end{tabular} & \begin{tabular}{|l}
0.22 \\
$(0.12,0.37)$
\end{tabular} & $\begin{array}{l}0.33 \\
(0.093,1.12)\end{array}$ & \begin{tabular}{|l}
0.26 \\
$(0.069,0.94)$
\end{tabular} & $\begin{array}{l}0.44 \\
(0.18,1.04)\end{array}$ & -- & -- \\
\hline $\begin{array}{l}0.82 \\
(0.21,2.85)\end{array}$ & \begin{tabular}{|l}
0.30 \\
$(\mathbf{0 . 0 8 2 , 0 . 9 8 )}$
\end{tabular} & $\begin{array}{l}\text { DC } \\
+ \text { FOLFOX }\end{array}$ & $\begin{array}{l}1.33 \\
(0.24,8.02)\end{array}$ & $\begin{array}{l}1.82 \\
(0.41,8.18)\end{array}$ & $\begin{array}{l}1.57 \\
(0.30,8.86)\end{array}$ & $\begin{array}{l}1.16 \\
(0.28,5.00)\end{array}$ & $\begin{array}{l}0.99 \\
(0.18,5.72)\end{array}$ & $0.76(0.12,5.06)$ & $\begin{array}{l}0.77 \\
(0.17,3.45)\end{array}$ & $\begin{array}{l}1.18 \\
(0.19,7.61)\end{array}$ & $\begin{array}{l}0.94 \\
(0.14,6.33)\end{array}$ & $\begin{array}{l}1.55 \\
(0.31,8.25)\end{array}$ & -- & -- \\
\hline $\begin{array}{l}0.77 \\
(0.21,2.42)\end{array}$ & \begin{tabular}{|l}
0.28 \\
$(0.083,0.80)$
\end{tabular} & $\begin{array}{l}0.94 \\
(0.17,5.09)\end{array}$ & $\begin{array}{l}\text { SM } \\
+ \text { FOLFOX }\end{array}$ & $\begin{array}{l}1.36 \\
(0.39,4.54)\end{array}$ & $\begin{array}{l}1.17 \\
(0.27,5.10)\end{array}$ & $\begin{array}{l}0.86 \\
(0.27,2.8)\end{array}$ & \begin{tabular}{|l}
0.74 \\
$(0.17,3.23)$
\end{tabular} & $\begin{array}{l}0.57 \\
(0.11,2.99)\end{array}$ & \begin{tabular}{|l}
0.58 \\
$(0.17,1.93)$
\end{tabular} & $\begin{array}{l}0.88 \\
(0.17,4.57)\end{array}$ & $\begin{array}{l}0.69 \\
(0.12,3.81)\end{array}$ & $\begin{array}{l}1.16 \\
(0.28,4.69)\end{array}$ & -- & -- \\
\hline $\begin{array}{l}1.004 \\
(0.58,1.73)\end{array}$ & \begin{tabular}{|l}
0.37 \\
$(0.26,0.52)$
\end{tabular} & $\begin{array}{l}1.23 \\
(0.35,4.72)\end{array}$ & \begin{tabular}{|l}
1.31 \\
$(0.43,4.65)$
\end{tabular} & $\begin{array}{l}\text { SQFZ } \\
+ \text { FOLFOX }\end{array}$ & $\begin{array}{l}0.87 \\
(0.28,2.79)\end{array}$ & \begin{tabular}{|l}
0.64 \\
$(0.32,1.32)$
\end{tabular} & \begin{tabular}{|l}
0.55 \\
$(0.17,1.80)$
\end{tabular} & $\begin{array}{l}0.42 \\
(0.11,1.68)\end{array}$ & \begin{tabular}{|l}
0.43 \\
$(0.19,0.94)$
\end{tabular} & $\begin{array}{l}0.66 \\
(0.17,2.55)\end{array}$ & $\begin{array}{l}0.52 \\
(0.12,2.12)\end{array}$ & $\begin{array}{l}0.86 \\
(0.30,2.46)\end{array}$ & -- & -- \\
\hline $\begin{array}{l}1.001 \\
(0.38,2.62)\end{array}$ & $\begin{array}{l}0.37 \\
(0.15,0.86)\end{array}$ & $\begin{array}{l}1.23 \\
(0.28,5.92)\end{array}$ & $\begin{array}{l}1.31 \\
(0.34,5.76)\end{array}$ & $\begin{array}{l}1.00 \\
(0.39,2.52)\end{array}$ & $\begin{array}{l}\text { DLS } \\
+ \text { FOLFOX }\end{array}$ & $\begin{array}{l}0.73 \\
(0.25,2.17)\end{array}$ & \begin{tabular}{|l}
0.63 \\
$(0.15,2.61)$
\end{tabular} & \begin{tabular}{|l}
0.49 \\
$(0.097,2.39)$
\end{tabular} & $\begin{array}{l}0.49 \\
(0.15,1.51)\end{array}$ & $\begin{array}{l}0.76 \\
(0.15,3.61)\end{array}$ & $\begin{array}{l}0.60 \\
(0.11,2.98)\end{array}$ & $\begin{array}{l}0.99 \\
(0.26,3.65)\end{array}$ & -- & -- \\
\hline $\begin{array}{l}1.24 \\
(0.73,2.15)\end{array}$ & \begin{tabular}{|l}
0.45 \\
$(0.32,0.64)$
\end{tabular} & $\begin{array}{l}1.53 \\
(0.44,5.85)\end{array}$ & \begin{tabular}{|l}
1.63 \\
$(0.53,5.71)$
\end{tabular} & $\begin{array}{l}1.24 \\
(0.75,2.02)\end{array}$ & $\begin{array}{l}1.25 \\
(0.49,3.18)\end{array}$ & $\begin{array}{l}\text { CKS } \\
+ \text { FOLFOX }\end{array}$ & $\begin{array}{l}0.85 \\
(0.28,2.59)\end{array}$ & $\begin{array}{l}0.66 \\
(0.17,2.48)\end{array}$ & \begin{tabular}{|l}
0.67 \\
$(0.33,1.32)$
\end{tabular} & $\begin{array}{l}1.026 \\
(0.27,3.76)\end{array}$ & $\begin{array}{l}0.81 \\
(0.20,3.15)\end{array}$ & $\begin{array}{l}1.35 \\
(0.50,3.54)\end{array}$ & -- & -- \\
\hline $\begin{array}{l}0.95 \\
(0.45,1.99)\end{array}$ & \begin{tabular}{|l}
0.35 \\
$(0.19,0.64)$
\end{tabular} & $\begin{array}{l}1.18 \\
(0.30,5.02)\end{array}$ & $\begin{array}{l}1.24 \\
(0.37,4.78)\end{array}$ & \begin{tabular}{|l}
0.95 \\
$(0.47,1.92)$
\end{tabular} & $\begin{array}{l}0.96 \\
(0.33,2.76)\end{array}$ & $\begin{array}{l}0.77 \\
(0.37,1.55)\end{array}$ & $\begin{array}{l}\text { HCS } \\
+ \text { FOLFOX }\end{array}$ & \begin{tabular}{|l}
0.77 \\
$(0.15,3.94)$
\end{tabular} & \begin{tabular}{|l}
0.78 \\
$(0.24,2.49)$
\end{tabular} & $\begin{array}{l}1.21 \\
(0.24,5.86)\end{array}$ & \begin{tabular}{|l}
0.94 \\
$(0.18,4.83)$
\end{tabular} & $\begin{array}{l}1.58 \\
(0.40,6.01)\end{array}$ & -- & -- \\
\hline $\begin{array}{l}0.44 \\
(0.12,1.48)\end{array}$ & \begin{tabular}{|l}
0.16 \\
$(0.049,0.50)$
\end{tabular} & \begin{tabular}{|l}
0.54 \\
$(0.096,3.13)$
\end{tabular} & $\begin{array}{l}0.58 \\
(0.12,3.07)\end{array}$ & $\begin{array}{l}0.44 \\
(0.13,1.45)\end{array}$ & $\begin{array}{l}0.44 \\
(0.10,1.86)\end{array}$ & \begin{tabular}{|l}
0.35 \\
$(0.10,1.16)$
\end{tabular} & \begin{tabular}{|l}
0.45 \\
$(0.12,1.71)$
\end{tabular} & $\begin{array}{l}\text { AP } \\
+ \text { FOLFOX }\end{array}$ & $\begin{array}{l}1.009 \\
(0.25,4.01)\end{array}$ & $\begin{array}{l}1.56 \\
(0.27,9.03)\end{array}$ & $\begin{array}{l}1.23 \\
(0.20,7.37)\end{array}$ & \begin{tabular}{|l}
2.042 \\
$(0.44,9.22)$
\end{tabular} & -- & -- \\
\hline $\begin{array}{l}1.18 \\
(0.69,2.04)\end{array}$ & $\begin{array}{l}0.43 \\
(0.31,0.6)\end{array}$ & $\begin{array}{l}1.46 \\
(0.42,5.53)\end{array}$ & $\begin{array}{l}1.55 \\
(0.52,5.39)\end{array}$ & $\begin{array}{l}1.18 \\
(0.73,1.91)\end{array}$ & $\begin{array}{l}1.19 \\
(0.47,3.03)\end{array}$ & $\begin{array}{l}0.95 \\
(0.59,1.54)\end{array}$ & $\begin{array}{l}1.24 \\
(0.62,2.49)\end{array}$ & \begin{tabular}{|l}
2.72 \\
$(0.82,9.30)$
\end{tabular} & $\begin{array}{l}\text { KA } \\
+ \text { FOLFOX }\end{array}$ & $\begin{array}{l}1.53 \\
(0.39,5.95)\end{array}$ & $\begin{array}{l}1.21 \\
(0.29,4.94)\end{array}$ & \begin{tabular}{|l}
2.011 \\
$(0.71,5.76)$
\end{tabular} & -- & -- \\
\hline $\begin{array}{l}0.80 \\
(0.21,2.76)\end{array}$ & \begin{tabular}{|l}
0.29 \\
$(0.082,0.94)$
\end{tabular} & $\begin{array}{l}0.97 \\
(0.17,5.77)\end{array}$ & $\begin{array}{l}1.051 \\
(0.20,5.51)\end{array}$ & $\begin{array}{l}0.79 \\
(0.22,2.7)\end{array}$ & $\begin{array}{l}0.79 \\
(0.18,3.43)\end{array}$ & \begin{tabular}{|l}
0.64 \\
$(0.18,2.20)$
\end{tabular} & $\begin{array}{l}0.83 \\
(0.20,3.13)\end{array}$ & $\begin{array}{l}1.79 \\
(0.35,9.78)\end{array}$ & \begin{tabular}{|l}
0.68 \\
$(0.18,2.25)$
\end{tabular} & $\begin{array}{l}\text { PL } \\
+ \text { FOLFOX }\end{array}$ & $\begin{array}{l}0.79 \\
(0.13,4.72)\end{array}$ & $\begin{array}{l}1.31 \\
(0.29,6.10)\end{array}$ & - & -- \\
\hline $\begin{array}{l}1.096 \\
(0.52,2.30)\end{array}$ & \begin{tabular}{|l}
0.40 \\
$(0.21,0.74)$
\end{tabular} & $\begin{array}{l}1.35 \\
(0.35,5.54)\end{array}$ & $\begin{array}{l}1.44 \\
(0.41,5.48)\end{array}$ & $\begin{array}{l}1.094 \\
(0.53,2.21)\end{array}$ & $\begin{array}{l}1.097 \\
(0.37,3.26)\end{array}$ & $\begin{array}{l}0.89 \\
(0.43,1.80)\end{array}$ & $\begin{array}{l}1.15 \\
(0.47,2.78)\end{array}$ & \begin{tabular}{|l}
2.52 \\
$(0.66,9.57)$
\end{tabular} & $\begin{array}{l}0.92 \\
(0.46,1.86)\end{array}$ & $\begin{array}{l}1.37 \\
(0.37,5.55)\end{array}$ & $\begin{array}{l}\text { LE } \\
+ \text { FOLFOX }\end{array}$ & $\begin{array}{l}1.66 \\
(0.35,8.12)\end{array}$ & -- & -- \\
\hline $\begin{array}{l}1.96 \\
(0.54,6.76)\end{array}$ & $\begin{array}{l}0.71 \\
(0.21,2.34)\end{array}$ & \begin{tabular}{|l}
2.42 \\
$(0.42,13.52)$
\end{tabular} & \begin{tabular}{|l}
2.57 \\
$(0.5,14.04)$
\end{tabular} & $\begin{array}{l}1.95 \\
(0.55,6.73)\end{array}$ & $\begin{array}{l}1.96 \\
(0.43,8.70)\end{array}$ & $\begin{array}{l}1.57 \\
(0.44,5.38)\end{array}$ & \begin{tabular}{|l}
2.04 \\
$(0.52,7.68)$
\end{tabular} & \begin{tabular}{|l}
4.42 \\
$(0.84,24.08)$
\end{tabular} & \begin{tabular}{|l}
1.64 \\
$(0.46,5.64)$
\end{tabular} & \begin{tabular}{|l}
2.46 \\
$(0.45,13.91)$
\end{tabular} & $\begin{array}{l}1.79 \\
(0.46,6.74)\end{array}$ & $\begin{array}{l}\text { XAP } \\
+ \text { FOLFOX }\end{array}$ & -- & -- \\
\hline $\begin{array}{l}1.25 \\
(0.34,4.77)\end{array}$ & $\begin{array}{l}0.46 \\
(0.13,1.61)\end{array}$ & $\begin{array}{l}1.54 \\
(0.28,9.59)\end{array}$ & $\begin{array}{l}1.66 \\
(0.33,9.30)\end{array}$ & $\begin{array}{l}1.24 \\
(0.34,4.60)\end{array}$ & $\begin{array}{l}1.24 \\
(0.28,5.90)\end{array}$ & $\begin{array}{l}1.006 \\
(0.28,3.76)\end{array}$ & $\begin{array}{l}1.31 \\
(0.34,5.28)\end{array}$ & \begin{tabular}{|l}
2.81 \\
$(0.55,16.15)$
\end{tabular} & \begin{tabular}{|l}
1.054 \\
$(0.30,3.86)$
\end{tabular} & $\begin{array}{l}0.64 \\
(0.11,3.40)\end{array}$ & $\begin{array}{l}0.88 \\
(0.21,3.44)\end{array}$ & $\begin{array}{l}1.53 \\
(0.26,9.29)\end{array}$ & $\begin{array}{l}\text { EL } \\
+ \text { FOLFOX }\end{array}$ & -- \\
\hline $\begin{array}{l}1.32 \\
(0.36,4.53)\end{array}$ & $\begin{array}{l}0.48 \\
(0.14,1.53)\end{array}$ & $\begin{array}{l}1.65 \\
(0.29,9.15)\end{array}$ & \begin{tabular}{|l}
1.73 \\
$(0.34,9.11)$
\end{tabular} & $\begin{array}{l}1.31 \\
(0.37,4.38)\end{array}$ & \begin{tabular}{|l}
1.31 \\
$(0.30,5.59)$
\end{tabular} & \begin{tabular}{|l}
1.061 \\
$(0.30,3.56)$
\end{tabular} & \begin{tabular}{|l}
1.38 \\
$(0.36,5.16)$
\end{tabular} & $\begin{array}{l}3.03 \\
(0.57,15.64)\end{array}$ & \begin{tabular}{|l}
1.11 \\
$(0.31,3.71)$
\end{tabular} & $\begin{array}{l}0.60 \\
(0.11,3.28)\end{array}$ & $\begin{array}{l}0.84 \\
(0.22,3.29)\end{array}$ & \begin{tabular}{|l}
1.48 \\
$(0.28,7.96)$
\end{tabular} & $1.075(0.18,5.48)$ & $\begin{array}{l}\text { GP } \\
+ \text { FOLFOX }\end{array}$ \\
\hline
\end{tabular}

differences were detected between these groups. Among CHIs groups, there was no significantly statistical difference between groups (Table 3). According to Figure 4C, the rank of 9 CHIs based on cumulative probabilities for hepatic dysfunction was namely: Disodium cantharidinate and vitamin B6 $(76.68 \%, 1$ trail $)>$ Shenqifuzheng $(75.83 \%$,
1 trail $)>$ Compound kushen $(70.54 \%, 6$ trails $)>$ Ginseng polysacchride $(68.73 \%, 1$ trail $)>$ Aidi $(66.51 \%, 3$ trails $)>$ Kangai $(50.49 \%, 3$ trails $)>$ Xiaoaiping $(30.99 \%, 2$ trails) $>$ Lentinan $(24.62 \%, 1$ trails $)>$ Shenmai $(22.26 \%, 1$ trail). In addition, the SUCRA values of each comparison for 5 outcomes were summarized in Table 4.

A
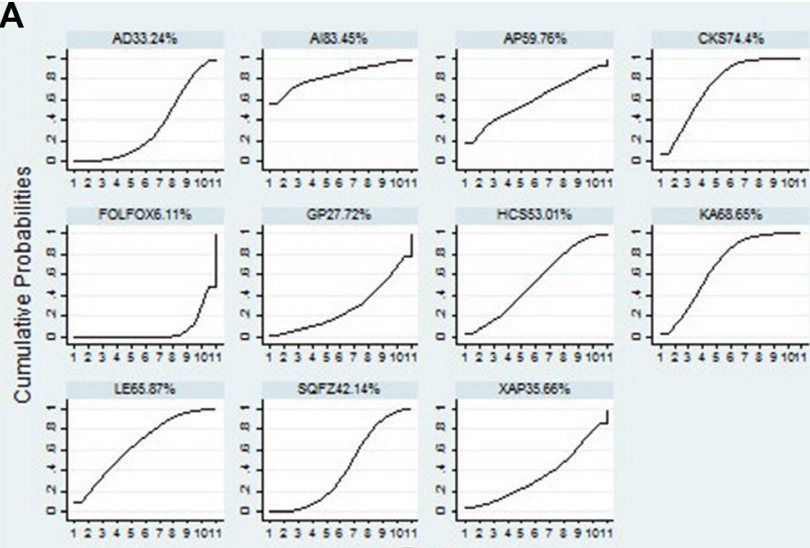

B
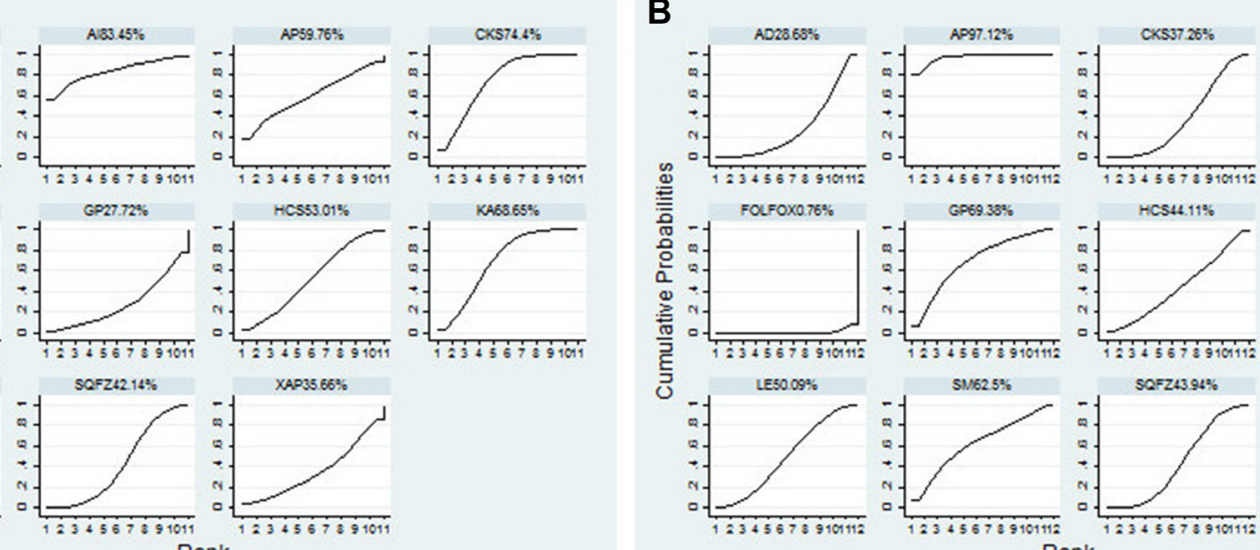

Graphs by Treatment

Rank
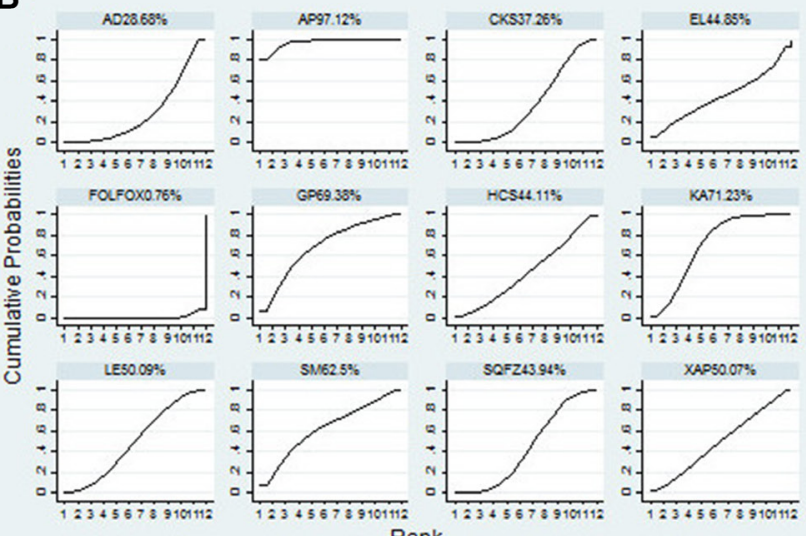

Graphs by Treatment

Rank

Figure 3: Rank of cumulative probabilities of clinical efficacy and performance status. Note: (A) Clinical efficacy; (B) Performance status; FOLFOX: FOLFOX Chemotherapy regimen; AD: Aidi injection; AI: Astragalus injection; AP: Astragalus polysaccharide injection; CKS: Compound kushen injection; GP: Ginseng Polysacchride Injection; HCS: Huachansu injection; KA: Kangai injection; LE: Lentinan injection; SQFZ: Shenqifuzheng injection; XAP: Xiaoaiping injection.EL: Elemene injection; SM: Shenmai injection. 
Table 3: Results of the network meta-analysis for hepatic dysfunction (lower left quarter)

\begin{tabular}{|c|c|c|c|c|c|c|c|c|c|}
\hline $\begin{array}{l}\text { AD+ } \\
\text { FOLFOX }\end{array}$ & & & & & & & & & \\
\hline $\begin{array}{l}4.38 \\
(1.76,11.08)\end{array}$ & FOLFOX & & & & & & & & \\
\hline $\begin{array}{l}0.53 \\
(0.016,6.48)\end{array}$ & $\begin{array}{l}0.12 \\
(0.0041,1.26)\end{array}$ & $\begin{array}{l}\text { DC+ } \\
\text { FOLFOX }\end{array}$ & & & & & & & \\
\hline $\begin{array}{l}3.69 \\
(0.62,21.1) \\
\end{array}$ & $\begin{array}{l}0.84 \\
(0.18,3.75) \\
\end{array}$ & \begin{tabular}{|l|}
7.01 \\
$(0.42,279.2)$ \\
\end{tabular} & \begin{tabular}{|l|} 
SM+ \\
FOLFOX \\
\end{tabular} & & & & & & \\
\hline $\begin{array}{l}0.64 \\
(0.052,5.16) \\
\end{array}$ & \begin{tabular}{l|}
0.15 \\
$(0.014,0.94)$ \\
\end{tabular} & \begin{tabular}{|l|}
1.19 \\
$(0.041,55.93)$ \\
\end{tabular} & \begin{tabular}{|l|}
0.17 \\
$(0.011,1.98)$ \\
\end{tabular} & \begin{tabular}{|l|} 
SQFZ+ \\
FOLFOX \\
\end{tabular} & & & & & \\
\hline $\begin{array}{l}0.93 \\
(0.30,2.72)\end{array}$ & $\begin{array}{l}0.21 \\
(0.11,0.38)\end{array}$ & $\begin{array}{l}1.71 \\
(0.15,52.25)\end{array}$ & $\begin{array}{l}0.25 \\
(0.048,1.28)\end{array}$ & $\begin{array}{l}1.45 \\
(0.20,16.1)\end{array}$ & $\begin{array}{l}\text { CKS+ } \\
\text { FOLFOX }\end{array}$ & & & & \\
\hline $\begin{array}{l}1.51 \\
(0.41,5.36) \\
\end{array}$ & \begin{tabular}{|l|}
0.34 \\
$(0.14,0.82)$ \\
\end{tabular} & \begin{tabular}{|l|}
2.78 \\
$(0.23,94.5)$ \\
\end{tabular} & \begin{tabular}{|l|}
0.41 \\
$(0.073,2.34)$ \\
\end{tabular} & \begin{tabular}{|l|}
2.39 \\
$(0.29,27.12)$ \\
\end{tabular} & $\begin{array}{l}1.63 \\
(0.56,4.77) \\
\end{array}$ & \begin{tabular}{|l|} 
KA+ \\
FOLFOX \\
\end{tabular} & & & \\
\hline $\begin{array}{l}0.86 \\
(0.10,5.96)\end{array}$ & $\begin{array}{l}0.20 \\
(0.029,1.09)\end{array}$ & \begin{tabular}{|l|}
1.60 \\
$(0.078,70.97)$
\end{tabular} & $\begin{array}{l}0.23 \\
(0.021,2.34)\end{array}$ & $\begin{array}{l}1.37 \\
(0.092,24.58)\end{array}$ & $\begin{array}{l}0.93 \\
(0.13,5.79)\end{array}$ & $\begin{array}{l}0.57 \\
(0.070,3.95)\end{array}$ & $\begin{array}{l}\text { GP+ } \\
\text { FOLFOX }\end{array}$ & & \\
\hline $\begin{array}{l}4.17 \\
(0.28,67.87) \\
\end{array}$ & $\begin{array}{l}0.96 \\
(0.077,12.81)\end{array}$ & \begin{tabular}{|l|}
8.066 \\
$(0.24,505.5)$ \\
\end{tabular} & $\begin{array}{l}1.13 \\
(0.061,23.5)\end{array}$ & \begin{tabular}{|l}
6.94 \\
$(0.27,196.2)$ \\
\end{tabular} & $\begin{array}{l}4.54 \\
(0.34,66.17)\end{array}$ & \begin{tabular}{|l|}
2.79 \\
$(0.20,43.73)$ \\
\end{tabular} & $\begin{array}{l}5.035 \\
(0.23,115.2)\end{array}$ & \begin{tabular}{|l} 
LE+ \\
FOLFOX \\
\end{tabular} & \\
\hline $\begin{array}{l}2.70 \\
(0.55,12.89)\end{array}$ & $\begin{array}{l}0.61 \\
(0.17,2.20)\end{array}$ & $\begin{array}{l}5.095 \\
(0.35,173.7)\end{array}$ & $\begin{array}{l}0.73 \\
(0.10,5.19)\end{array}$ & $\begin{array}{l}4.27 \\
(0.44,59.85)\end{array}$ & $\begin{array}{l}2.90 \\
(0.72,11.84)\end{array}$ & $\begin{array}{l}1.79 \\
(0.38,8.37)\end{array}$ & $\begin{array}{l}3.13 \\
(0.37,32.46)\end{array}$ & $\begin{array}{l}0.64 \\
(0.036,10.63)\end{array}$ & $\begin{array}{l}\text { XAP+ } \\
\text { FOLFOX }\end{array}$ \\
\hline
\end{tabular}

\section{Publication bias}

The publication bias of the included RCTs were measured by funnel plots. The funnel plots of 5 outcomes in this NMA illustrated there were potential publication bias among included RCTs (Figure 5).

\section{Cluster analysis}

The cluster analysis based on SUCRA values indicated that interventions with same color belonged to the same cluster, and interventions located in the upper right corner seemed an optimal therapy for two different outcomes. The results of cluster analysis were revealed in Figure 6. First, a cluster analysis was conducted for 9 types of CHIs that reported both clinical efficacy, and performance status. Astragalus polysaccharides + FOLFOX and Kangai +FOLFOX showed a favorable improvement of clinical efficacy and performance status for patients with gastric cancer. While Aidi +FOLFOX was inferior in improving the primary outcomes among CHIs groups, and receiving FOLFOX regimen single was proved to be the worst intervention. Second, the results of cluster analysis which accessed 12 CHIs for leucopenia and gastrointestinal reaction indicated that Astragalus polysaccharides +FOLFOX was associated with a better effect on relieving both leucopenia and gastrointestinal reaction, by contrast, Xiaoaiping +FOLFOX exhibited an inferior response for these ADRs among CHIs groups. Third, 8 types of CHIs contributed into the cluster analysis for leucopenia and hepatic dysfunction. The therapies of Disodium cantharidinate and vitamin B6 +FOLFOX, Aidi +FOLFOX, Compound +FOLFOX were associated with preferable response in relieving leucopenia and hepatic dysfunction. Similarly, for gastrointestinal reaction and hepatic dysfunction, Disodium cantharidinate and vitamin B6 +FOLFOX also achieve superior effects for reducing both gastrointestinal reaction and hepatic dysfunction among CHIs groups.
A

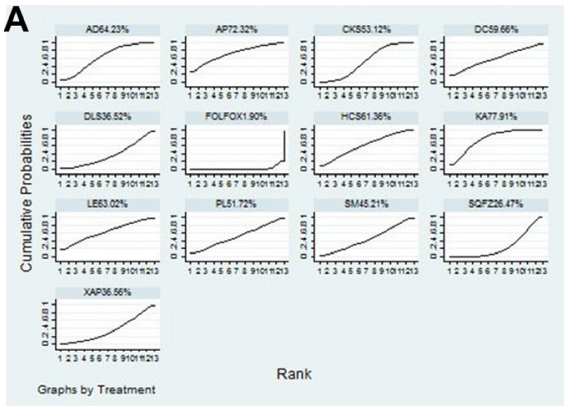

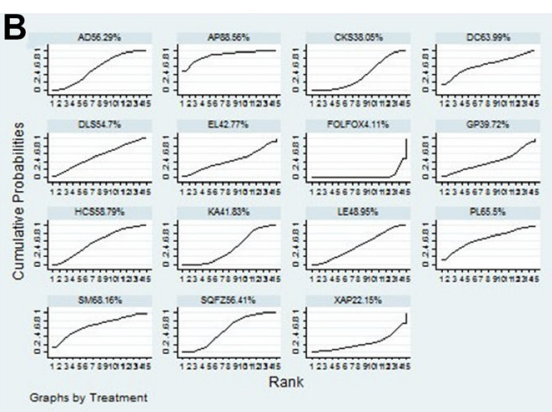

Figure 4: Rank of cumulative probabilities of ADRs. Note: (A) Leucopenia; (B) Gastrointestinal reaction; (C) Hepatic dysfunction; FOLFOX:FOLFOX Chemotherapy regimen; AD: Aidi injection; AP: Astragalus polysaccharide injection; CKS: Compound kushen injection; DC: Disodium cantharidinate and vitamin B6 injection; DLS: Delisheng injection; HCS: Huachansu injection; KA: Kangai injection; LE: Lentinan injection; PL: Placenta polypeptide injection; SM: Shenmai injection; SQFZ: Shenqifuzheng injection; XAP: Xiaoaiping injection; EL: Elemene injection; GP: Ginseng Polysacchride Injection. 
Table 4: SUCRA values of 15 CHIs groups and FOLFOX group for 5 outcomes

\begin{tabular}{|c|c|c|c|c|c|}
\hline & $\begin{array}{c}\text { The clinical } \\
\text { efficacy }\end{array}$ & $\begin{array}{c}\text { Performance } \\
\text { status }\end{array}$ & Leukopenia & $\begin{array}{c}\text { Gastrointestinal } \\
\text { reaction }\end{array}$ & $\begin{array}{c}\text { Hepatic } \\
\text { dysfunction }\end{array}$ \\
\hline $\mathrm{AD}+\mathrm{FOLFOX}$ & $33.24 \%$ & $28.68 \%$ & $64.23 \%$ & $56.29 \%$ & $66.51 \%$ \\
\hline FOLFOX & $6.11 \%$ & $0.76 \%$ & $1.90 \%$ & $4.11 \%$ & $13.36 \%$ \\
\hline $\mathrm{AI}+\mathrm{FOLFOX}$ & $83.45 \%$ & NR & NR & NR & NR \\
\hline $\mathrm{AP}+\mathrm{FOLFOX}$ & $59.76 \%$ & $97.12 \%$ & $72.32 \%$ & $88.56 \%$ & NR \\
\hline $\mathrm{CKS}+\mathrm{FOLFOX}$ & $74.4 \%$ & $37.26 \%$ & $53.12 \%$ & $38.05 \%$ & $70.54 \%$ \\
\hline $\mathrm{DC}+\mathrm{FOLFOX}$ & NR & NR & $59.66 \%$ & $63.99 \%$ & $76.68 \%$ \\
\hline DLS + FOLFOX & NR & NR & $36.52 \%$ & $54.7 \%$ & NR \\
\hline $\mathrm{EL}+\mathrm{FOLFOX}$ & NR & $44.85 \%$ & NR & $42.77 \%$ & NR \\
\hline GP + FOLFOX & $27.72 \%$ & $69.38 \%$ & NR & $39.72 \%$ & $68.73 \%$ \\
\hline HCS + FOLFOX & $53.01 \%$ & $44.11 \%$ & $61.36 \%$ & $58.79 \%$ & NR \\
\hline $\mathrm{KA}+\mathrm{FOLFOX}$ & $68.65 \%$ & $71.23 \%$ & $77.91 \%$ & $41.83 \%$ & $50.49 \%$ \\
\hline $\mathrm{LE}+\mathrm{FOLFOX}$ & $65.87 \%$ & $50.09 \%$ & $63.02 \%$ & $48.95 \%$ & $24.62 \%$ \\
\hline PL + FOLFOX & NR & NR & $51.72 \%$ & $65.5 \%$ & NR \\
\hline $\mathrm{SM}+\mathrm{FOLFOX}$ & NR & $62.5 \%$ & $45.21 \%$ & $68.16 \%$ & $22.26 \%$ \\
\hline $\mathrm{SQFZ}+\mathrm{FOLFOX}$ & $42.14 \%$ & $43.94 \%$ & $26.47 \%$ & $56.41 \%$ & $75.83 \%$ \\
\hline XAP + FOLFOX & $35.66 \%$ & $50.07 \%$ & $36.56 \%$ & $22.15 \%$ & $30.99 \%$ \\
\hline
\end{tabular}

Note: NR:nor report; FOLFOX: FOLFOX Chemotherapy regimen; AD: Aidi injection; AI: Astragalus injection; AP: Astragalus polysaccharide injection; CKS: Compound kushen injection; DC: Disodium cantharidinate and vitamin B6 injection; DLS: Delisheng injection; EL: Elemene injection; GP: Ginseng Polysacchride Injection; HCS: Huachansu injection; KA: Kangai injection; LE: Lentinan injection; PL: Placenta polypeptide injection; SM: Shenmai injection; SQFZ: Shenqifuzheng injection; XAP: Xiaoaiping injection.

A
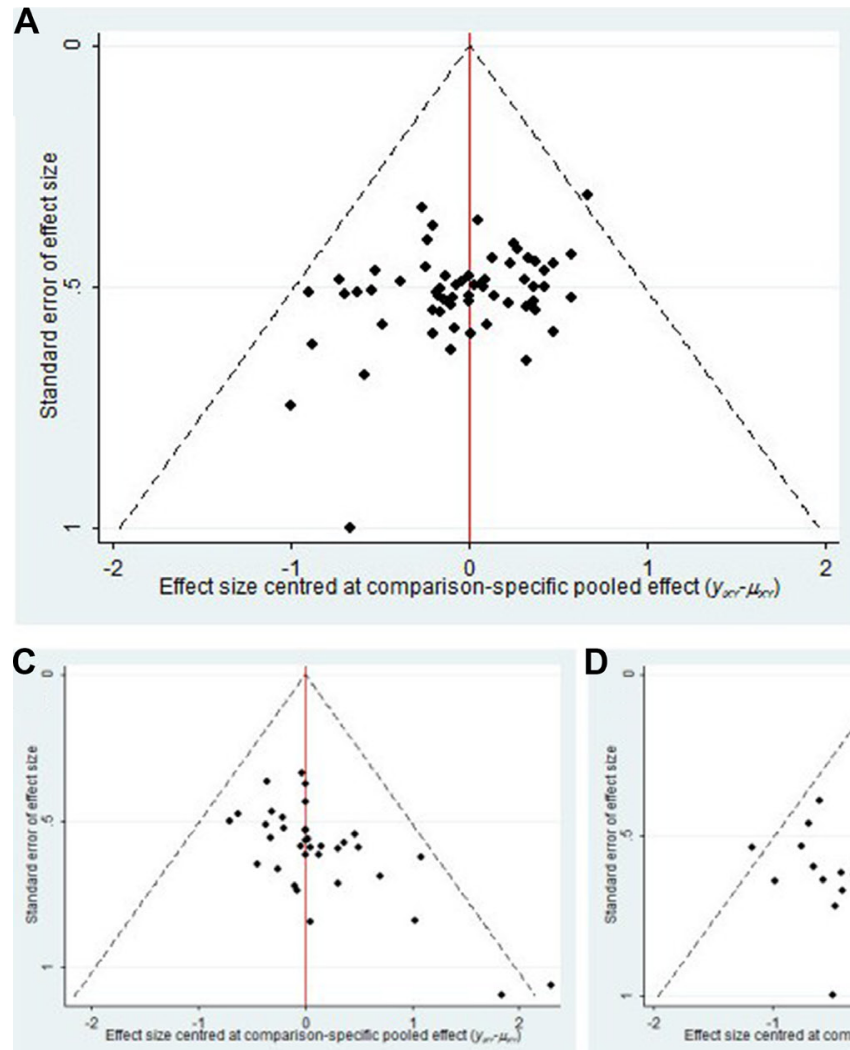

B
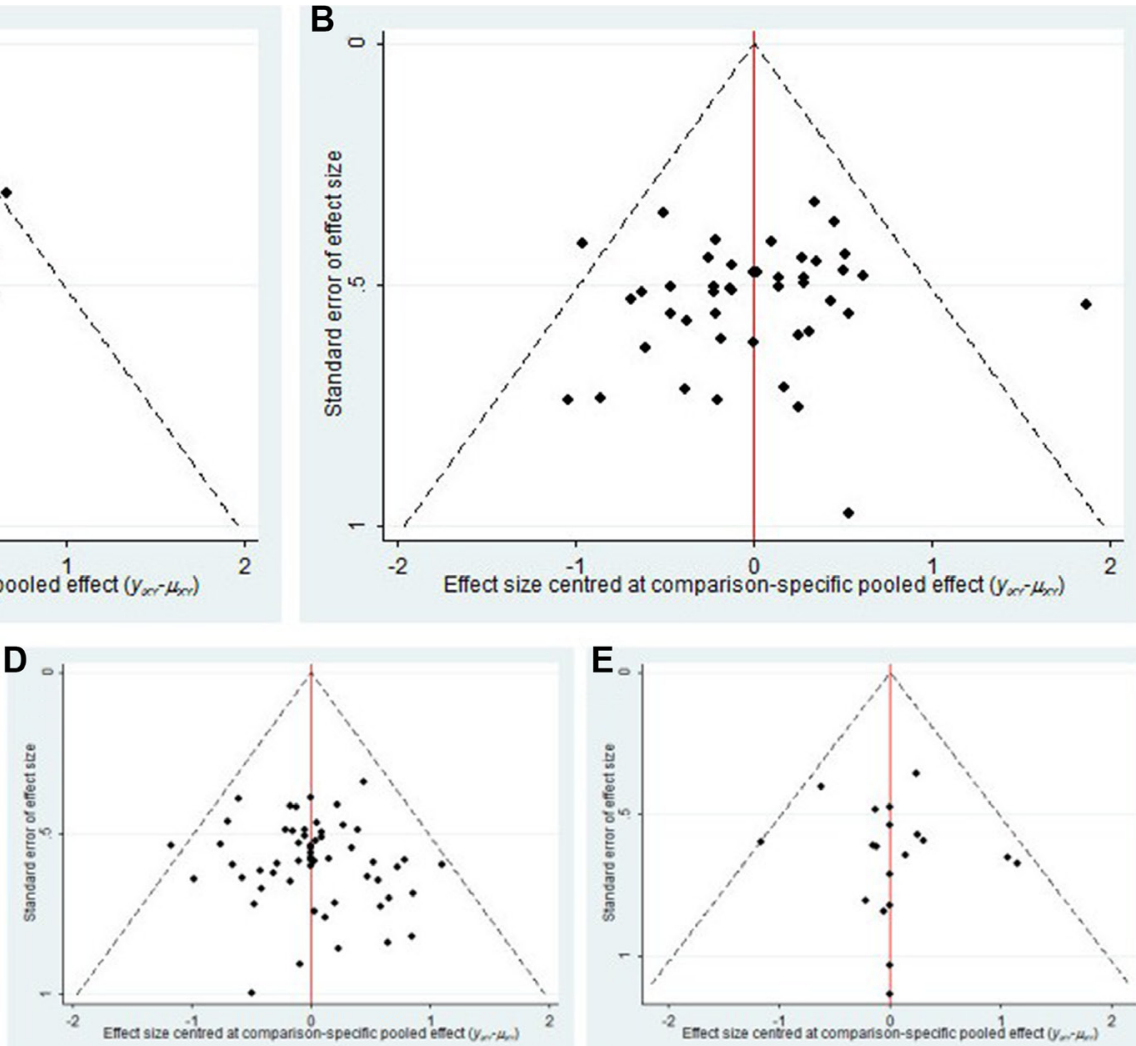

Figure 5: Funnel Plot for 5 outcomes. Note: (A) Clinical efficacy; (B) Performance status; (C) Leucopenia; (D) Gastrointestinal reaction; (E) Hepatic dysfunction. 


\section{DISCUSSION}

This NMA was conducted to compare 15 types of CHIs combined with FOLFOX regimen for the treatment of gastric cancer with regard to efficacy and safety. According to the results of this NMA, we suggested that Astragalus polysaccharides injection and Kangai injection combined with FOLFOX regimen were associated with preferable effects in improving clinical efficacy and performance status. In terms of ADRs, Astragalus polysaccharides injection and Disodium Cantharidinate and Vitamin B6 injection could achieve a favorable response in relieving ADRs. In general, different types of CHIs had different effects and functions on patients with gastric cancer [107]; therefore, the clinical decision of using CHIs should depend on the combination of doctors' experience, patients' condition and high-level evidencebased medical research.
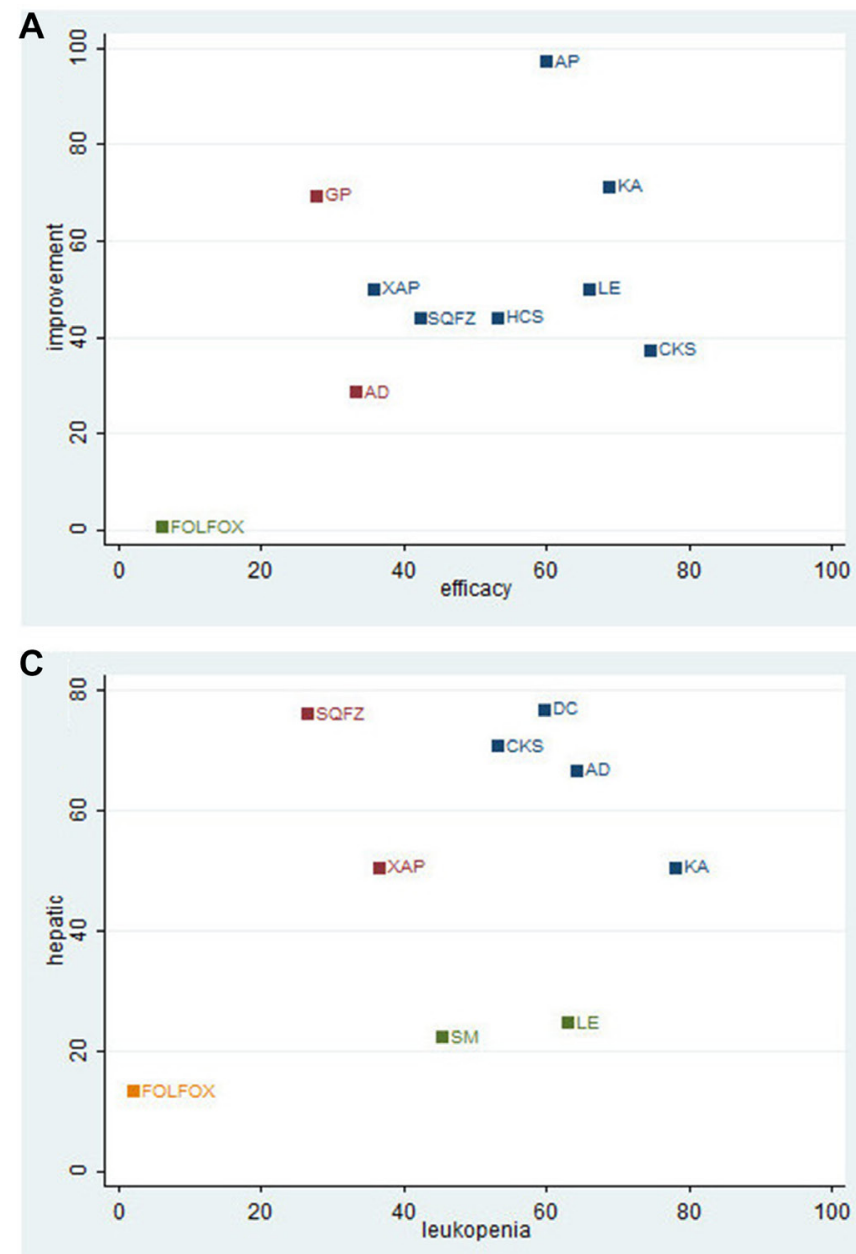

In view of clinical manifestation, gastric cancer belonged to the category of "stomachache", "dysphagia" and "nausea" in TCM theory [108]. Recently, TCM served as important parts of complementary and alternative medicine to provide a theoretical and practical approach to the treatment of gastric cancer combined with other conventional cancer therapies. And TCM was confirmed that it could not only improve the clinical symptoms, immune functions and performance status of cancer patients, but also prolong their survival period [109-110]. Moreover, Kangai injection was made from ginseng, Astragalus, Sophora flavescens, and its functions were replenishing Qi and strengthening the body resistance owing to its active components, namely Astragalus saponins, ginsenoside, and matrine. Correlative pharmacological studies reported that Kangai injection might influence the enzyme activities of macrophages and morphology for spleen and thymus from rats
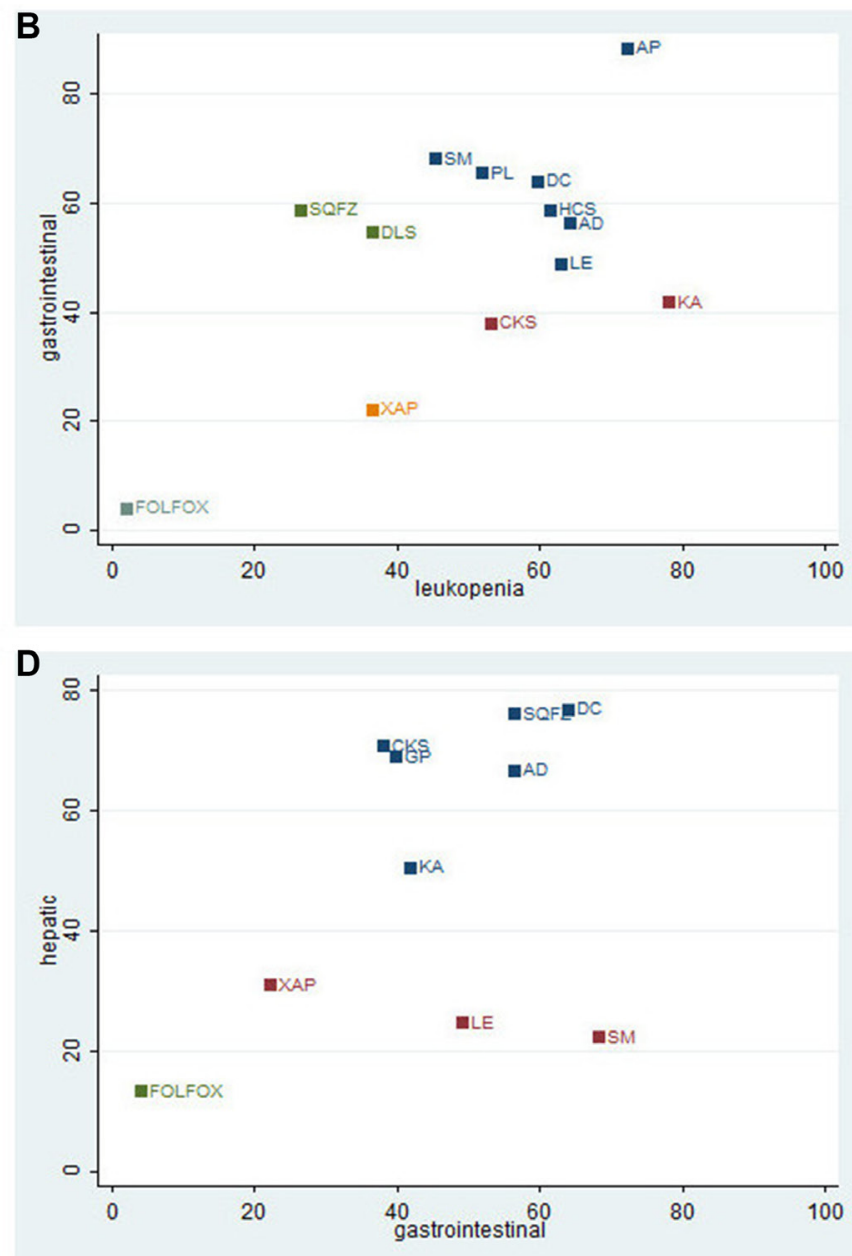

Figure 6: Cluster analysis Plot for 5outcomes. Note: (A) Clinical efficacy (X axis) \& Performance status (Y axis); (B) Leukopenia (X axis) \& Gastrointestinal reaction (Y axis); (C) Leukopenia (X axis) \& Hepatic dysfunction (Y axis); (D) Gastrointestinal reaction (X axis) \& Hepatic dysfunction (Y axis); FOLFOX: FOLFOX Chemotherapy regimen; AD: Aidi injection; CKS: Compound kushen injection; SQFZ: Shenqifuzheng injection; HCS: Huachansu injection; XAP: Xiaoaipinginjection; LE: Lentinan injection; GP: Ginseng Polysacchride Injection; KA: Kangai injection; AP: Astragalus polysaccharide injection. DLS: Delisheng injection; DC: Disodium cantharidinate and vitamin B6 injection; PL: Placenta polypeptide injection; SM: Shenmai injection. 
[111]. Meanwhile, Kangai injection combined with chemotherapy could achieve effects for treating malignant pleural effusion and for refractory/relapsed acute leukemia [112-113]. From the theory of TCM, the flavor of Astragalus mongholicus was sweet; the nature of it was slightly warm, and its actions included supplement and boost Qi and lifting Yang. Modern pharmacological studies also showed that different Astragali radix extracts had antineuropathic effects of oxaliplatin-induced neurotoxicity in a rat model [114]. On the one hand, Astragalus saponins could inhibit the growth both in vitro and vivo of human gastric cancer cells; on the other hand, Astragalus saponins were able to reduce the invasion ability and induce the apoptosis of gastric cancer cells [115]. In addition, it was proven that Astragalus polysaccharide can effectively alleviate inflammation and boost the immune system to achieve the strong effects of antitumor [116-117]. Meanwhile, some clinical studies revealed that Astragalus polysaccharide injection combined chemotherapy could improve the clinical efficacy and prolong the survival time of the patients with nonsmall-cell lung cancer [118].

FOLFOX regimen waas accepted as one of the firstline chemotherapy regimen for the treatment of gastric cancer internationally $[7,10]$. The chemotherapy regimen of included RCTs was restricted to FOLFOX regimen for reducing the interference of clinical heterogeneity which was related to chemotherapeutic agents. Currently, there was one research of NMA regarding CHIs for treating gastric cancer [119], compared with this previous research, the advantages of this NMA were reflected in the following aspects: Firstly, the searching strategy was more comprehensive because that literature searches were conducted about 23 types of CHIs which were used for treating cancer at the present, and the searching strategy was amended and confirmed by experts on data retrieval methods. Besides, apart from searching electronic databases of Chinese and English, we also searched RCTs at related academic organization websites. Secondly, the inclusion and exclusion criteria were established strictly. The standardization of efficacy evaluation was only according to WHO criteria. Third, the results of our NMA was in accordance with the previous research in Astragalus polysaccharide injection could achieve a superior response for clinical efficacy and safety than other CHIs; however, with regard to the results of Kanglaite injection and Javanica oil emulsion injection, the conclusions were not totally consistent between this NMA and the previous research. Finally, we not only accessed the clinical efficacy, performance status and ADRs, but also evaluated the quality and estimated the publication bias of included RCTs; the cluster analysis was also conducted to select the best CHIs for different outcomes.

Also, this NMA faced several limitations inherent to the methodology applied. First of all, survival time was an important end-point outcome for evaluating the efficacy in the treatment of cancer. While only 4 RCTs in this NMA provided the sufficient data of survival time or follow-up as showed in Supplementary Table 1. Therefore, the data was not enough to perform a NMA for the outcome of survival time. Second, our results were limited by methodological quality of the included RCTs; the majority of included RCTs exhibited a high risk of bias, largely due to inadequate allocation concealment and blinding methods. Among 15 CHIs involved in this NMA, 6 types of CHIs only had 1 trial included. The trial bias might affect the comparison objectivity between the different CHIs. And there was lack of large sample-size trails and head-to-head comparisons which was conducted between different CHIs. Third, although CHIs were widely applied in China, the test population of CHI was subject to other countries and regions. And the included RCTs were conducted in China and published in Chinese; therefore, it is unclear whether the conclusions of our study apply to other populations. Given those shortcomings, our research were needed to confirm by large-sample and multicenter, head-to-head RCTs. Moreover, our research suggested that clinical trials should concentrate on improving methodological quality themselves, adopt more objective, international endpoints outcomes to provide more rigorous and reliable evidence for clinical decision-making. In spite of those limitations listed above, this NMA provided a clear rank and optimal options by comparing clinical efficacy, performance status, and ADRs of different CHIs combined with FOLFOX regimen for treating gastric cancer.

The results of this NMA suggested that among 15 types of CHIs, Astragalus polysaccharides injection combined with FOLFOX regimen seemed optimal for patients with gastric cancer in improving clinical efficacy and performance status, and relieving ADRs. However, our findings should be confirmed by more prospectively designed, large-sample and multi-center RCTs.

\section{MATERIALS AND METHODS}

This systematic and NMA was conducted in accordance with Cochrane criteria and PRISMA guidelines.

\section{Search strategy}

From inception to January 10, 2017, an extensive literature collection about RCTs regarding CHIs for treating gastric cancer was performed by comprehensive searching strategy. The electronic databases included PubMed, Embase, the Cochrane Library, and together with several Chinese databases: the China National Knowledge Infrastructure Database (CNKI), the Wan-Fang Database (WangFang), the Chinese Scientific Journals Full-text Database (VIP), and the Chinese Biomedical Literature Database (CBM). Literature management was conducted by using EndNote X7 software (Thomson Reuters 
Crp.3 Times Square, New York, The United States). The keywords about gastric cancer of PubMed yielded the following searching query: "Stomach Neoplasm, Stomach Neoplasm, Gastric Neoplasms, Gastric Neoplasm, Stomach Cancer*, Stomach Tumor*, Gastric Cancer*, Gastric Tumor*, Gastric Carcinoma, Stomach Carcinoma". And the Chinese keywords about gastric cancer in this NMA were according to the mesh terms which were provided in CBM database. The specific search terms for each CHIs and retrieval strategies were shown in Supplementary Table 3.

\section{Selection criteria}

Four researchers (DZ, JZ, MN, and $\mathrm{WJ}$ ) took participate in making the inclusion and exclusion criteria of this NMA.

Only RCTs meeting the following criteria were included: (1) Participant: the included participants diagnosed as gastric cancer, and no gender, race, or nationality limitations were imposed. (2) Intervention/ Control: The CHIs group was treated by $\mathrm{CHIs}$ combined with FOLFOX regimen, while FOLFOX group solely received FOLFOX regimen. The chemotherapeutic drugs of FOLFOX regimen included 5-Fu, LV and L-OHP. (3) Outcomes: The primary outcomes were the clinical efficacy and the performance status. The criterion of the rapeutical effect met the WHO for solid tumors released in 1979 [120].The clinical efficacy was calculated as followings: the clinical efficacy $=[$ number of complete response $(\mathrm{CR})$ patients + partial response $(\mathrm{PR})] /$ total number of patients $\times 100 \%$. Performance status was assessed by KPS, patients were considered to improve performance status when their KPSs increased more than 10 points after treatment. The secondary outcomes were the ADRs involving the incidence of leucopenia, gastrointestinal reaction and hepatic dysfunction. And the criterion of the ADRs met the WHO for common toxicity criteria of chemotherapy drugs released in 1981 [121]. The incidence of ADRs = (number of patients occurred ADRs $) /$ total number of patients $\times 100 \%$. (4) Study type: RCTs regarding CHIs were combined with FOLFOX regimen for the treatment of gastric cancer, with irrespective of blinding methods the publishing language.

The exclusion criteria were described as follows: (1) Participant: Patients were complicated by other cancers, contraindications of chemotherapy and obvious abnormalities in their electrocardiograms and liver and kidney functions. (2) Intervention/Control: The administration of CHIs was not intravenous infusion. And information about chemotherapeutic drugs, dose and duration of treatment was incomplete or incorrect. Except for FOLFOX regimen, patients also received by radiotherapy, hyperthermia, interventional therapy, etc. (3) Outcomes: there were not available data of clinical efficacy, performance status and ADRs, and the rapeutical effect or ADRs was not in accordance with the criterion of WHO. (4) Study type: the publication types were not RCTs; such as case reports, animal experiments, editorials, letters, and reviews. And for duplicated RCTs, only the most updated and comprehensive ones were chosen.

\section{Data extraction and quality assessment}

The available data of included RCTs was extracted into a spreadsheet of Microsoft Excel (Microsoft Corp, Redmond, WA) by three researchers (DZ, JZ, MN), and two researchers (KW, XD) crosschecked the extracted data independently. The following items were extracted: (1) Study characteristics: title, authors' names, publication year, and literature sources of RCTs; (2) Patient characteristics: the numbers, ages, genders, KPSs before treatment, tumor types, and tumor stages of patients; (3) Intervention information: the names, dosages, and treatment cycles of CHIs; (4) Outcomes: the measured data of clinical efficacy, performance status and ADRs. Additionally, more details about the product information of each CHI were presented in Supplementary Table 4.

Quality assessment (including the randomization method, follow-up, blind methods, allocation concealment, reasons for withdrawal, inclusion and exclusion criteria, adverse reactions, statistical methods, foundations, and medical ethics) was performed by two independent researchers (DZ, JW), and disagreements were resolved by consensus according to the Cochrane risk of bias tool [122] and the methodological section of the CONSORT statement [123].

\section{Statistical analysis}

The odds ratios (OR) of dichotomous data was calculated to measure comparative efficacy and safety for each therapy, along with $95 \%$ confidence intervals (CI). All the results of OR value were presented as medians. STATA 12.0 software (Stata Corporation, College Station, TX, USA) was adopted to present all calculations and graphs of NMA, and Markov chain Monte Carlo methods were performed by Win-BUGS 1.4.3 software (MRC Biostatistics Unit, Cambridge, UK). First, the chi-squared test was used to evaluate heterogeneity among studies, and $\mathrm{I}^{2}$ was used to show the magnitude of this heterogeneity. Results of $P \geq 0.1$ and $\mathrm{I}^{2} \leq 50 \%$ suggested a lack of significant heterogeneity; in such cases, the MantelHaenszel fixed-effects model was chosen. For cases with $P<0.1$ and $\mathrm{I}^{2}>50 \%$, the Mantel-Haenszel random-effects model should be applied [124-125]. Since taking into account the included trails differed methodologically and clinically, the random-effects model was introduced to perform this NMA[126]. Second, we used surface under the cumulative ranking probabilities (SUCRA) values to rank the examined treatments, with SUCRA values of $100 \%$ and $0 \%$ assigned to the best and worst 
treatments [127-128]. Third, publication bias was graphically accessed via a comparison-adjusted funnel plot. Furthermore, we utilized clustering methods and 2-dimensional plots to produce clusters of treatments. Evaluation of the inconsistency between direct and indirect comparisons was unnecessary because a loop connecting the three arms did not exist in this NMA.

\section{Abbreviations}

CHIs: Chinese herbal injections; RCTs: Randomized controlled trials; NMA: network meta-analysis; ADR: adverse reaction; NMA = network meta-analysis; OR: odds ratios; CI: confidence interval; 5-Fu: 5-fluorouracil, LV: leucovorin, L-OHP: oxaliplatin; TCM: traditional Chinese medicine; CNKI: the China National Knowledge Infrastructure Database; VIP: the Chinese Scientific Journals Full-text Database; CBM: the Chinese Biomedical Literature Database; WHO: World Health Organization; KPS: Karnofsky performance score; SUCRA: surface under the cumulative ranking probabilities; AD: Aidi injection; DC: Disodium cantharidinate and vitamin B6 injection; SM: Shenmai injection; SQFZ: Shenqifuzheng injection; DLS: Delisheng injection; CKS: Compound kushen injection; HCS: Huachansu injection; AP: Astragalus polysaccharide injection; AI: Astragalus injection; KA: Kangai injection; EL: Elemene injection; GP: Ginseng Polysacchride injection; PL: Placenta polypeptide injection; LE: Lentinan injection; XAP: Xiaoaiping injection.

\section{Author contributions}

Conception and design of the experiments: DZ, JW. Performance of the experiments: DZ, JZ, MN, JW, KW, XD. Analysis of study data: DZ, JZ,MN, JW. Contribution of reagents/materials/analysis tools: DZ, MZ. Writing of the paper: DZ, MN, JW. Quality control for the study: JW, BZ.

\section{ACKNOWLEDGMENTS}

The authors are very appreciative of the entire participants for their support and help in the paper editing.

\section{CONFLICTS OF INTEREST}

The authors declare that they have no competing interests.

\section{FUNDING}

This research is supported by the National Natural Science Foundation of China (No. 81473547), the National Natural Science Foundation of China (No. 81673829).

\section{REFERENCES}

1. Torre LA, Bray F, Siegel RL, Ferlay J, Lortet-Tieulent J, Jemal A. Global cancer statistics, 2012. CA Cancer J Clin. 2015; 65:87-108. https://doi.org/10.3322/caac.21262.

2. Siegel R, Ma J, Zou Z, Jemal A. Cancer statistics, 2014. CA Cancer J Clin. 2014; 64:9-29. https://doi.org/10.3322/ caac. 21208 .

3. Chen W, Zheng R, Baade PD, Zhang S, Zeng H, Bray F, Jemal A, Yu XQ, He J. Cancer statistics in China, 2015. CA Cancer J Clin. 2016; 66:115-32. https://doi.org/10.3322/caac.21338.

4. Abedi-Ardekani B,Hainaut P. Cancers of the Upper Gastro-intestinal Tract: A Review of Somatic Mutation Distributions. Arch Iran Med. 2014; 17:286-292. https:// doi.org/014174/AIM.0012.

5. Jin ML. Progress of systemic chemotherapy for advanced gastric cancer. Oncology Progress. 2007; 5:18.

6. Ren J, Kuang TH, Chen J, Yang JW, Liu YX. The diagnostic and prognostic values of microRNA-21 in patients with gastric cancer: a meta-analysis. Eur Rev Med Pharmacol Sci. 2017; 21:120-130.

7. National Health and Family Planning Commission of the People's Republic of China Guidelines for standardized diagnosis and treatment of gastric cancer (Trial Implementation). Chinese Journal of The Frontiers of Medical Science. 2013; 5:29-36.

8. Brenkman HJ, Haverkamp L, Ruurda JP, van Hillegersberg R. Worldwide practice in gastric cancer surgery. World $\mathrm{J}$ Gastroenterol. 2016; 22:4041-4048.

9. Jácome AA, Coutinho AK, Lima EM, Andrade AC, Dos Santos JS. Personalized medicine in gastric cancer: Where are we and where are we going? World J Gastroenterol. 2016 ; 22:1160-1171.

10. Ajani JA, D'Amico TA, Almhanna K, Bentrem DJ, Chao J, Das P, Denlinger CS, Fanta P, Farjah F, Fuchs CS, Gerdes H, Gibson M, Glasgow RE, et al. Gastric Cancer, Version 3.2016, NCCN Clinical Practice Guidelines in Oncology. J Natl Compr Canc Netw. 2016; 14:1286-1312.

11. Yamamoto S, Ono H, Kume K, Ohsawa M. Oxaliplatin treatment changes the function of sensory nerves in rats. J Pharmacol Sci. 2016; 130:189-193.

12. Wang B, Walsh SJ, Saif MW. Pancytopenia and Severe Gastrointestinal Toxicities Associated with 5-Fluorouracil in a Patient with Thymidylate Synthase (TYMS) Polymorphism. Cureus. 2016; 8:e798.

13. Qi F, Li A, Inagaki Y, Gao J, Li J, Kokudo N, Li XK, Tang W. Chinese herbal medicines as adjuvant treatment during chemo- or radio-therapy for cancer. Biosci Trends. 2010; 4:297-307.

14. Chen Z, Wang P. Clinical Distribution and Molecular Basis of Traditional Chinese Medicine ZHENG in Cancer. Evid Based Complement Alternat Med. 2012; 2012:783923.

15. Zhang J, Zhan Z, Wu J, Zhang C, Yang Y, Tong S, Wang R, Yang X3, Dong W, Chen Y. Relationship between EGF, 
TGFA, and EGFR Gene Polymorphisms and Traditional Chinese Medicine ZHENG in Gastric Cancer. Evid Based Complement Alternat Med. 2013; 2013:731071.

16. Hong M, Wang N, Tan HY, Tsao SW, Feng Y. MicroRNAs and Chinese Medicinal Herbs: New Possibilities in Cancer Therapy. Cancers (Basel). 2015; 7:1643-1657.

17. Konkimalla VB, Efferth T. Evidence-based Chinese medicine for cancer therapy. J Ethnopharmacol. 2008; 116:207-210.

18. Li G, Jian H, Yang YN, Tian M, Yang X, Zhu CH, Ding XL, Liu XH, Zhang HR, Liu C, Qin XM, Wu XZ. Network pharmacology dissection of multiscale mechanisms of herbal medicines in stage IV gastric adenocarcinoma treatment. Medicine (Baltimore). 2016; 35:e4389.

19. Dai X, Liu D, Liu M, Zhang X, Wang W, Jin F, Qian Y, Wang X, Zhao J, Wu Y, Xiong F, Chang NA, Sun YU, et al. Anti-metastatic efficacy of traditional Chinese medicine (TCM) ginsenoside conjugated to a VEFGR-3 antibody on human gastric cancer in an orthotopic mouse model. Anticancer Res. 2017; 3:979-986.

20. Rasul A, Yu B, Yang LF, Ali M, Khan M, Ma T, Yang H. Induction of mitochondria-mediated apoptosis in human gastric adenocarcinoma SGC-7901 cells by kuraridin and Nor-kurarinone isolated from Sophora flavescens. Asian Pac J Cancer Prev. 2011; 10:2499-504.

21. Wu M, Lu P, Shi L, Li S. Traditional Chinese patent medicines for cancer treatment in China: a nationwide medical insurance data analysis. Oncotarget. 2015; 6:38283-95. https://doi. org/10.18632/oncotarget.5711.

22. Luo D, Ye LH. Present situation and trend of Chinese medicine in the treatment for liver cancer at experimental research field. World Chin J Digestology. 2008; 16:2964-68.

23. Jansen JP, Naci H. Is network meta-analysis as valid as standard pairwise meta-analysis? It alldepends on the distribution of effect modifiers. BMC Med. 2013; 11:159.

24. Lumley T. Network meta-analysis for indirect treatment comparisons. Stat Med. 2002; 21: 2313-2324.

25. Migliore A, Broccoli S, Massafra U, Bizzi E, Frediani B. Mixed-treatment comparison of anabolic(teriparatide and PTH 1-84)therapies in women with severe osteoporosis. Curr Med Res Opin. 2012; 28:467-473.

26. Wu T, Li RC. The efficacy and toxicity of FOLFOX7 regimen combined with Aidi injection in the treatment of advanced gastric cancer. Guangdong Yixue. 2016; 37:438-440.

27. Liao YQ, Tong WW, Zhan WH. The short-term efficacy of Aidi injection combined with FOLFOX4 regimen for treating advanced gastric cancer. Journal of Ningxia Medical University. 2011; 33:668-669.

28. Zhang L. Study of Aidi injection combined with FOLFOX4 chemotherapy for advanced gastric cancer. Cardiovascular Disease Journal of Integrated Traditional Chinese and Western Medicine (Electronic). 2014; 49-50.

29. Chen NJ, Wu DH, Lai YQ, Chen YY. Combining AIDI, FOLFOX4 treated advanced gastric cancer. Guang Ming Journal of Chinese Medicine. 2008; 23:1768-1769.
30. Song SJ, Li SY, Zhao DH, Bai LY, Guo K. Clinical value of Aidi injection combined with FOLFX4 in the treatment of advanced gastric cancer. Chinese Journal of Trauma and Disability Medicine. 2014; 153-153.

31. He CS. Curative effect observation of Aidi injection combined with chemotherapy in the treatment of advanced gastric cancer. Guide of China Medicine. 2012; 10:492-493.

32. Yan HX, Yang GW, Wang ZH, Ye YI. Therapeutic efficacy of Aidi Injection combined with chemotherapy for gastric cancer and effect of it on immunological function. China Pharmacy. 2012; 3392-3394.

33. Zhang AX. Observation of curative effect for Aidi injection on advanced gastric cancer. The Journal of Medical Theory and Practice. 2009; 22:1214-1215.

34. Zhang MJ, Zuo CF. The clinical efficacy of Aidi injection in patients with advanced gastric cancer. Guide of China Medicine. 2014; 190-191.

35. Li GP, Li XR, Wang JH. Clinical observation on sodium cantharidate vitamin B6 injection combined with postoperative chemotherapy in treatment of gastric cancer. Pract Prev Med. 2010; 17:105-106.

36. Zhang SQ, Li LF, Zheng HB, Fang MM. Clinical Observation of Advanced Gastric Cardia Cancer Treated with Shenmai Injection Combined with Chemotherapy. Guide of China Medicine. 2013; 12-13.

37. Song SQ. Effects of Shenqifuzheng injection on immune function in patients with advanced gastric cancer undergoing chemotherapy. Med Infant. 2015; 5:299-300.

38. Liu H, Han D. Effects of Shenqifuzheng injection on the immunolologic function and clinic efficacy in the elderly patients with advanced gastric cancer. Zhongguo Lin Chuang Yao Li Xue Za Zhi. 2011; 27:177-179.

39. Xie YF, Fu RY, Guan XJ. Influence of Shenqifuzheng injection on efficacy of chemotherapy and immune function in patients with gastric cancer after surgery. Chinese Journal of Primary Medicine and Pharmacy. 2015; 22:3078-3081.

40. Xu XG, Wang D, Huang Y, Huang YJ. Clinical observation on treatment of 31 cases of advanced gastric cancer (deficiency of spleen and stomach) with Shenqifuzheng injection. Guiding Journal of Traditional Chinese Medicine and Pharmacy. 2015; 32-35.

41. $\mathrm{Pa} \mathrm{TM}, \mathrm{Gu}$ LZ. Therapeutic effect of Shenqifuzheng injection combined with FOLFOX4 on advanced gastric cancer. World Latest Medicine Information. 2012; 46-47.

42. Wang M, Lin HJ. Efficacy observation of Shenqi Fuzheng injection combined with FOLFOX4 chemotherapy in treatment of advanced gastric cancer. China Modern Medicine. 2011;18:89-90.

43. Fang XY. Observation of efficacy of Shenqi Fuzheng injection combined with FOLFOX4 chemotherapy in treatment of advanced gastric cancer. Chinese Journal of Practical Medicine. 2010; 37:23-24.

44. Jia JW, Liu YQ. The Effect of Shenqifuzheng injection combined with FOLFOX4 regimen in the treatment of advanced gastric cancer. Practical Journal of Cancer. 2009; 24:273-275. 
45. Wang P. Effect of Shenqifuzheng injection combined with chemotherapy on the clinical efficacy and immune function in patients with advanced gastric cancer. Clin Med (Northfield III). 2014; 34:124-125.

46. Ren YZ, Wang F. Influence of advanced gastric cancer treated with Shenqifuzheng injection combined with chemotherapy on effect and immune function. Journal of Basic and Clinical Oncology. 2012; 25:394-396.

47. Sun YF, Pei Y, Tian YX, Liu YH. Therapeutic effect of Shenqifuzheng injection combined with chemotherapy on advanced gastric cancer in patients with Qi deficiency. Proceedings of the Eighth National Conference on Gastropathy. 2008:166-169.

48. Wen J.Clinical observation of advanced gastric cancer treated with Shenqi fuzheng injection combined with chemotherapy. Yangzhou University. 2014.

49. Li HY, Ji WM, Tu J, Shen YL. Clinical analysis of Shenqi Fuzheng Injection combined with chemotherapy in patients with advanced gastric cancer. China Medical Herald. 2013; 10:99-101.

50. Lai CH, Wang SC, Tian RF, Liu SY. Clinical observation and nursing of Shenqifuzheng injection combined with chemotherapy in the treatment of advanced gastric cancer. Health must-Read magazine. 2013; 12:227-228.

51. Chen LL, Jin LX, Mao L, Yan HF, Feng CZ. Clinical observation of Shenqifuzheng injection combined with chemotherapy in the treatment of advanced gastric cancer. Strait Pharmaceutical Journal. 2012; 24:111-113.

52. Zhu LF, Zhang YS, Wu Q. Clinical observation of Shenqi Fuzheng Injection Combined with chemotherapy in the treatment of gastric cancer. Shanghai Medical Journal. 2007; 30:542-543.

53. Wan LX, Wang Y. Treatment of advanced gastric cancer with Shenqifuzheng injection combined with FolFox4 regimen. Journal of Medical Forum. 2006; 27:96-97.

54. Huang D, Wang XD, Duan JP. Therapeutic effect of Shenqifuzheng injection combined with chemotherapy on 48 cases of gastric cancer. Modern Digestion \& Intervention. 2014; 324-326.

55. He ZQ, Cheng DY, Gong H, Yan H. The clinical efficacy of FOLFOX4 chemotherapy combined with Delisheng in therapy of advanced gastic cancer. Journal of Modern Oncology. 2008; 16:408-411.

56. Lu XF. Clinical efficacy and feasibility analysis of compound matrine injection combined with chemotherapy in the treatment of gastric cancer. Med Infant. 2015; 28:78-79.

57. Lv XY. Clinical value of compound matrine injection combined with FOLFOX4 regimen in the treatment of advanced gastric cancer. Journal of North Pharmacy. 2012; 9:17-18.

58. Zhang LQ, Tian SQ, Yu YH, Jin Y. Compound matrine injection in combination with FOLFOX4 regimen for gastric cancer: a clinical study. Zhongguo Yiyuan Yongyao Pingjia Yu Fenxi. 2010; 10:1030-31.
59. Tan HB, Zhang J, Zhang JW. Treatment of advanced gastric cancer with composed kushen injection combined with FOLFOX4 adjuvant chemotherapy. Wuhan Daxue Xuebao Yixue Ban. 2012; 33:215-18.

60. Liu KH, Wang YZ. Clinical observation of compound matrine injection combined with FOLFOX4 chemotherapy in the treatment of advanced gastric cancer. Asia-Pacific Traditional Medicine. 2014; 10:103-104.

61. Zhao Y. Clinical observation of compound kushen injection combined with chemotherapy in improving the life quality of patients with gastric cancer. Shandong University of Traditional Chinese Medicine. 2011.

62. Luo W. Compound matrine injection combined with chemotherapy in the treatment of advanced gastric cancer. Med Infant. 2014; 12:265-266.

63. Han QL, Zhang LF, Li J, Yang CM, Tang HL. Curative effect observation of Compound Matrine Injection combined with chemotherapy in treatment of gastric cancer. China Medical Herald. 2011; 68-69.

64. Liu SL, Ding C, Gu XX. Clinical observation on 29 cases of advanced gastric cancer treated with compound matrine injection combined with chemotherapy. Chin J Coal Ind Med. 2009; 12:1566-67.

65. Song SJ, Shen JH, Zhao DH, Li SY, Bai LY, Guo K, Zhang XA. Efficacy of compound matrine injection combined with chemotherapy in the treatment of gastric cancer. World Health Digest. 2013; 9:189.

66. Feng XM, Fu FJ, Han WQ, Feng XZ. Efficacy of compound matrine injection combined with chemotherapy in the treatment of advanced gastric cancer. National Medical Frontiers of China. 2013; 63-64.

67. Yang XE, Ye JK. Compound matrine injection combined with chemotherapy foe 50 cases of advanced gastric cancer. Zhongguo Zhongyiyao Xinxi Zazhi. 2013; 20:80-81.

68. Huang ZF, Liu JB, Li Hz, Huang CJ. Clinical observation on 30 cases of advanced gastric cancer treated with compound matrine injection combined with chemotherapy. Proceedings of the 21 st Academic Conference on digestive system diseases of traditional Chinese and Western Medicine. 2009:498-500.

69. Yang JW, Jiang SJ, Chen J. Adjuvant effect of compound matrine injection in postoperative chemotherapy of gastric cancer. Chinese Journal of Traditional Medical Ssience and Technology. 2012; 19:251-252.

70. Chen XQ,Liu NN, Shen DL. Study of compound matrine injection on preventing hepatic lesion caused by chemotherapy. Cancer Research and Clinic. 2010; 22:261-263.

71. Liu YH, Liu YH. Clinical observation on treatment of senile gastric cancer with Yanshu injection adjuvant. Jilin Medical Journal. 2010; 31:5332.

72. Xu JX, Wang AR, Wang WJ. Effect of Yanshu injection combined with chemotherapy on quality of life in patients with advancedgastric cancer. Chinese Journal of Clinical Oncology and Rehabilitation. 2013; 20:1043-1046. 
73. Zhang Y, Zhu M, Cao Y, Zhang P, Yao LG. Curative effect Observation of Huachansu injection combined treatment with chemotherapy on middle and advanced gastric cancer. Henan Journal of Oncology. 2005;18:359-360.

74. Wang ZF, Wang P. Observation on curative effect for Huachansu injection combined treatment with chemotherapy of advanced gastric cancer. Chinese Journal of Primary Medicine and Pharmacy. 2012; 19:1991-1992.

75. Lu CH, Hong M, Liu KH, Liu J. Clinical observation of Huachansu injection neoadjuvant chemotherapy for advanced gastric cancer. Traditional Chinese Medicine Journal. 2014; 13:41-43,49.

76. Cui P. Clinical observation on Huachansu injection the treatment of advanced gastric cancer with Chinese herb. Journal of Liaoning Medical University. 2009; 30:333-334.

77. Wang YH. Treatment of 36 cases of advanced gastric cancer with combination of Huachansu injection and chemotherapy. Chiang-Hsi Chung I Yao. 2009; 40:31-32.

78. Wang WM, Li CF, Yao RJ. Clinical observation on treatment of advanced gastric cancer by combined Huachansu injection and chemotherapy. Clinical Journal of Traditional Chinese Medicine. 2010; 22:314-315.

79. $\mathrm{Hu} \mathrm{HR}, \mathrm{Hu}$ JD. Efficacy of Astragalus injection combined with chemotherapy in patients with gastric cancer. Seek Medical and Ask the Medicine. 2012; 10:815-816.

80. Lai YB. Evaluation the clinical efficacy of Astragalus polysaccharide injection combined with chemotherapy in patients with advanced gastric cancer. For All Health. 2014; 8:162-162.

81. Liu YH, Huang J, Wang Y, Zheng ZS. Efficacy of Astragalus polysaccharide injection combined with chemotherapy in the treatment of advanced gastric cancer. The Journal of Practical Medicine. 2011; 27:516-518.

82. Li SQ.Analysis for the safety and feasibility of Kang' ai injection combined with FLO chemotherapy in treatment of advanced gastric cancer. Hebei Medical Journal. 2015; 37:1144-1147.

83. Yang XM. Kang'ai injection combined with FLO chemotherapy in the treatment of advanced gastric cancer: a clinical observation of 40 cases. Zhongguo Shiyan Fangjixue Zazhi. 2012; 18:325-327.

84. Zhou WJ, Wu J, Zhu DX, Wang Q, Zhang HY. Clinical observation of Kang'ai injection combined with FLO regimen in the treatment of 80 cases with advanced gastric cancer. Modern Diagnosis \& Treatment. 2015; 26:4840 4842 .

85. Li YY. To observe the curative effect of Kangai injection combined with FOLFOX4 regimen in the treatment of advanced gastric cancer. Journal of New Chinese Medicine. 2008; 40:23-24.

86. Qi YJ. The curative effect observation on changes of VEGF in serum level for Kang'ai injection combined with
FOLFOX4 regimen in the treatment of advanced gastric cancer. Zhongguo Zhongyao Zazhi. 2008; 33:2051-2052.

87. Wang JH. Clinical observation on Kang'ai injection combined with OLF regimen in the treatment of advanced gastric cancer. China Health Industry. 2011; 8:35.

88. Mo YY. Clinical analysis of aidi injection combined with oxaliplatin, leucovorin and fluorouracil in the treatment of advanced gastric cancer. Journal of Chinese Practical Diagnosis and Therapy. 2010; 24:1008-1009.

89. Wang LJ, Li XD, Zhang ZH. Clinical study of Kang'ai injection combined with chemotherapy in advanced gastric cancer. Zhongguo Zhongyiyao Xiandai Yuancheng Jiaoyu. 2008; 6:358-359.

90. Wu L, Yang Y. A clinical study of treating advanced gastric cancer with the combination of Kangai injection and chemotherapy. Proceeding of Clinical Medicine. 2009; 18:493-496.

91. Wang XQ, Wang DZ. Clinical observation of Kangai Injection combined with chemotherapy in treatment of gastric cancer. Liaoning Zhongyiyao Daxue Xuebao. 2013; $15: 210-212$.

92. Jiang L, Wang SQ, Yu D. Clinical study of FOLFOX4 regimen in the treatment of advanced gastric cancer with Kang'ai injection. Health World. 2013; 11:160-161.

93. Huang FJ, Li WY, Ma J. Research on improving the application value of elderly patients with advanced gastric carcinoma Kang'ai injection. China Health Industry. 2013; 9:76-77.

94. Leng S, Guo LY, Ren F. Research on the value of Kang'ai injection on gastric cancer patients' chemotherapy. China Medical Equipment. 2015; 12:134-136.

95. Su XH. Clinical observation of advanced gastric cancer by chemotherapy combined with OLF the effect of Kang'ai injection. Journal of Qiqihar University of Medicine. 2013; 34:2687-2688.

96. Zeng DX, Bi YZ, Ling Y, Yang QL. Clinical research of elemene emulsion combined with FOLFOX 4 regimen for advanced gastric cancer. Linchuang Zhongliuxue Zazhi. 2011; 16:917-919.

97. Xu JT. Effect of ginseng polysaccharide injection combined with chemotherapy on immune function and therapeutic of the patients with advanced gastric cancer .Central South Pharmacy. 2015; 13:316-318.

98. Zhang JX, Chen XQ, Zhang L. Clinical observation of Ginseng Polysacchride Injection combined with chemotherapy in treatment of advanced gastric cancer. Hebei Medical Journal. 2011; 33:1889.

99. Zhao LL, Zhang T, Fu PD, Wang RF, Tao N. Effect of placenta polypeptide injection combined with chemotherapy on quality of life in patients with gastric cancer. Journal of Harbin Medical University. 2014; 4:310-313.

100. Chang YF, Tang AM,Wang JF, Ge MD. Curative effect observation of lentinan for late gerontal gastric cancer and intestinal cancer. Modern Journal of Integrated Traditional 
Chinese and Western Medicine. 2008; 17:4375-4376.

101. Wu ZY, Huang WB. Analysis of efficacy of letinous edodes polysaccharide combined with chemotherapy in treatment of advanced gastric cancer. Med Infant. 2013; 7:253.

102. Wang HM, Liao GQ, Liu PH, Xie GQ, Qu YM. Lentinas Combined with FOLFOX4 Regimen in the Treatment of 68 Cases with Advanced Stomach Cancer. J Oncol. 2009; 15:333-334.

103. Li XL, Niu M, Zhang M, Zhang RQ. Effect of Lentinan Injection combined chemotherapy on immune function and clinical efficacy in patients with advanced gastric cancer. Shaanxi Medical Journal. 2015; 44:1624-1626.

104. Li YY. Treatment of 36 cases of gastric cancer with Lentinan Injection combined with chemotherapy. Shaanxi Journal of Traditional Chinese Medicine. 2010; 31:12-13.

105. Sai FD,Ma LY, Tang Y. Clinical Observation of Xiaoaiping Injection Combined with Chemotherapy on the Treatment of Advanced Gastric Cancer. Journal of Basic and Clinical Oncology. 2012; 25:397-399.

106. Liu HD, Zhu ZY. Study of Xiaoaiping Injection Combined with Chemotherapy on Treatment of Advanced Gastric Cancer. Hebei Med. 2012; 18:1704-1707.

107. Bai XJ. Analysis of antineoplastic varieties of traditional Chinese medicine injection. Pharmacology and Clinics of Chinese Materia Medica. 2009; 25:72-73.

108. Wang XL, Shi XW. Analysis of traditional Chinese medicine treatment strategies for gastric cancer. Chinese Journal of Chinese Medicine. 2012: 394-95.

109. Qi F, Zhao L, Zhou A, Zhang B, Li A, Wang Z, Han J. The advantages of using traditional Chinese medicine as an adjunctive therapy in the whole course of cancer treatment instead of only terminal stage of cancer. Biosci Trends. 2015; 9:16-34.

110. Mao Y, Hao J, Jin ZQ, Niu YY, Yang X, Liu D, Cao R, Wu XZ. Network pharmacology-based and clinically relevant prediction of the active ingredients and potential targets of Chinese herbs in metastatic breast cancer patients. Oncotarget. 2017; 8:27007-21. https://doi.org/10.18632/ oncotarget.15351.

111. Chen HB, Ying LL, Zhao LL, Xiang YX, Lin KZ, Wang W. The effects of Kangai injection on enzyme activities of macrophages in rats. Zhongguo Ying Yong Sheng Li Xue Za Zhi. 2014; 30:417-20.

112. Gu Y, Jiang L, Miao JH, Liang TS, Kan QC, Yang DK. Clinical effects of thermotherapy in combination with intracavitary infusion of traditional Chinese medicine in the treatment of malignant pleural effusion. J Biol Regul Homeost Agents. 2016; 30:1023-1028.

113. Wan Q, Xi A, Zhang C, Liu X. Clinical study of kangai injection plus FLAG regimen for refractory/relapsed acute leukemia. Zhongguo Zhongyao Zazhi. 2011; 36: 3207-3209.
114. Chinese Pharmacopoeia Commission. Pharmacopoeia of the People's Republic of China. China Medical Science Press. 2015; 1:302-3.

115. Di Cesare Mannelli L1 Pacini A, Micheli L, Femia AP, Maresca M, Zanardelli M, Vannacci A, Gallo E, Bilia AR, Caderni G, Firenzuoli F, Mugelli A, Ghelardini C. Astragali radix: could it be an adjuvant for oxaliplatininduced neuropathy? Sci Rep. 2017; 7:42021. https://doi. org/10.1038/srep42021.

116. Wang T, Xuan X, Li M, Gao P, Zheng Y, Zang W, Zhao G. Astragalus saponins affect proliferation, invasion and apoptosis of gastric cancer BGC-823 cells. Diagn Pathol. 2013; 8:179.

117. Cao Y, Ruan Y, Shen T, Huang X, Li M1, Yu W, Zhu Y, Man Y, Wang S, Li J. Astragalus polysaccharide suppresses doxorubicin-induced cardiotoxicity by regulating the PI3k/ Akt and p38MAPK pathways. Oxid Med Cell Longev. 2014; 2014:674219.

118. Guo L, Bai SP, Zhao L, Wang XH. Astragalus polysaccharide injection integrated with vinorelbine and cisplatin for patients with advanced non-small cell lung cancer: effects on quality of life and survival. Med Oncol. 2012; 29: 1656-62. https://doi.org/10.1007/s12032-011-0068-9.

119. Wang JC, Tian JH, Ge L, Gan YH, Yang KH. Which is the best Chinese herb injection based on the FOLFOX regimen for gastric cancer? A network meta- analysis of randomized controlled trials. Asian Pac J Cancer Prev. 2014; 15:4795-4800.

120. Miller AB, Hoogstraten B, Staquet M, Winkler A. Reporting results of cancer treatment. Cancer. 1981; 1:207-14.

121. People's Republic of China Department of Health Management. Guidelines for the diagnosisand treatment of cancer. Beijing: Pecking Union Medical College Press. 1991:11-15.

122. Higgins JPT, Green S. Cochrane Handbook for Systematic Reviews of Interventions Version 5.1.0 [updated March 2011]. The Cochrane Collaboration, 2011. Available from http://handbook.cochrane.org.

123. Moher D, Hopewell S, Schulz KF, Montori V, Gøtzsche PC, Devereaux PJ, Elbourne D, Egger M, Altman DG; Consolidated Standards of Reporting Trials Group. CONSORT 2010 explanation and elaboration: updated guidelines for reporting parallel group randomised trials. J Clin Epidemiol. 2010; 63:e1-37.

124. Higgins JP, Thompson SG, Deeks JJ, Altman DG. Measuring inconsistency in meta-analyses. BMJ. 2003; 327:557-60.

125. Salanti G, Higgins JP, Ades AE, Ioannidis JP. Evaluation of networks of randomized trials. Stat Methods Med Res. 2008; 17:279-301.

126. Tian JH, Li L, Yang KH. The realization of network Meta analysis of frequency statistics method in STATA software. Chinese Journal of Evidence Based Pediatrics. 2014; 9:472-474. 
127. Lunn DJ, Thomas A, Best N, Wakefield J, Spiegelhalter D. WinBUGS-a Bayesian modeling framework: concepts, structure, and extensibility. Stat Comput. 2000; 10:325-37.

128. Cope S, Jansen JP. Quantitative summaries of treatment effect estimates obtained with network meta-analysis of survival curves to inform decision-making. BMC Med Res Methodol. 2013; 13:147. 